\title{
Application of polymersomes engineered to target p32 protein for detection of small breast tumors in mice
}

\author{
Lorena Simón-Gracia ${ }^{1}$, Pablo Scodeller ${ }^{1}$, Sergio Salazar Fuentes ${ }^{2}$, Vanessa Gómez \\ Vallejo ${ }^{3}$, Xabier Ríos ${ }^{3}$, Eneko San Sebastián ${ }^{3}$, Valeria Sidorenko ${ }^{1}$, Desirè Di Silvio ${ }^{4}$, \\ Meina Suck ${ }^{5,6}$, Federica De Lorenzi ${ }^{5}$, Larissa Yokota Rizzo ${ }^{5}$, Saskia von Stillfried ${ }^{5}$, \\ Kalle Kilk7, Twan Lammers ${ }^{5,6}$, Sergio E. Moya ${ }^{4}$ and Tambet Teesalu ${ }^{1,8,9}$ \\ ${ }^{1}$ Laboratory of Cancer Biology, Institute of Biomedicine and Translational Medicine, University of Tartu, 50411 Tartu, \\ Estonia \\ ${ }^{2}$ Animal Unit, CIC Biomagune, 20009 Donostia, Spain \\ 3 Laboratory of Radiochemistry, CIC Biomagune, 20009 Donostia, Spain \\ ${ }^{4}$ Soft Matter Laboratoy, CIC Biomagune, 20009 Donostia, Spain \\ ${ }^{5}$ Institute for Experimental Molecular Imaging, RWTH Aachen University Clinic, 52074 Aachen, Germany \\ ${ }^{6}$ Department of Targeted Therapeutics, MIRA Institute for Biomedical Technology and Technical Medicine, University of \\ Twente, 7500 AE Enschede, The Netherlands \\ ${ }^{7}$ Department of Biochemistry, Institute of Biomedicine and Translational Medicine, University of Tartu, Tartu, 50411, \\ Estonia \\ ${ }^{8}$ Cancer Research Center, Sanford Burnham Prebys Medical Discovery Institute, La Jolla, California 92097, USA \\ ${ }^{9}$ Center for Nanomedicine, University of California Santa Barbara, Santa Barbara, California 93106, USA \\ Correspondence to: Tambet Teesalu, email: tteesalu@sbpdiscovery.org \\ Sergio E. Moya, email: smoya@cicbiomagune.es \\ Lorena Simón-Gracia, email: Lorena.Simon.Gracia@ut.ee
}

Keywords: PET; tumor-penetrating peptide; polymersomes; triple negative breast cancer; p32

Received: November 22, $2017 \quad$ Accepted: February 10, $2018 \quad$ Published: April 10, 2018

Copyright: Simón-Gracia et al. This is an open-access article distributed under the terms of the Creative Commons Attribution License 3.0 (CC BY 3.0), which permits unrestricted use, distribution, and reproduction in any medium, provided the original author and source are credited.

\section{ABSTRACT}

Triple negative breast cancer (TNBC) is the deadliest form of breast cancer and its successful treatment critically depends on early diagnosis and therapy. The multicompartment protein p32 is overexpressed and present at cell surfaces in a variety of tumors, including TNBC, specifically in the malignant cells and endothelial cells, and in macrophages localized in hypoxic areas of the tumor. Herein we used polyethylene glycol-polycaprolactone polymersomes that were affinity targeted with the p32-binding tumor penetrating peptide LinTT1 (AKRGARSTA) for imaging of TNBC lesions. A tyrosine residue was added to the peptide to allow for ${ }^{124} \mathrm{I}$ labeling and PET imaging. In a TNBC model in mice, systemic LinTT1-targeted polymersomes accumulated in early tumor lesions more than twice as efficiently as untargeted polymersomes with up to $20 \%$ ID/ $\mathrm{cc}$ at $24 \mathrm{~h}$ after administration. The PET-imaging was very sensitive, allowing detection of tumors as small as $\sim 20 \mathbf{~ m m}^{3}$. Confocal imaging of tumor tissue sections revealed a high degree of vascular exit and stromal penetration of LinTT1-targeted polymersomes and co-localization with tumor-associated macrophages. Our studies show that systemic LinTT1-targeted polymersomes can be potentially used for precision-guided tumor imaging and treatment of TNBC. 


\section{INTRODUCTION}

TNBC is defined by the lack of expression of estrogen receptor, progesterone receptor, and human epidermal growth factor 2 . TNBC accounts for $\sim 15 \%$ of all breast cancer cases and is a heterogeneous group of tumors that can be classified in different subtypes based on gene expression profiles [1-3]. Some TNBC subtypes respond to the chemotherapy and have favorable prognosis, whereas other TNBC subtypes are aggressive with average cancer recurrence within 3 years after initial diagnosis and a life expectancy of $\sim 5$ years [4]. These aggressive TNBC are locally invasive, highly metastatic, and must be detected and treated early to prevent dissemination.

Nanoformulations offer unique advantages for drug delivery. Nanoparticles can be designed to encapsulate hydrophobic molecules that would otherwise be insoluble, and payloads that have short circulation half-life and/or need to be protected from enzymes in the bloodstream, such as esterases or nucleases [5]. Cancer diagnosis and treatment can be combined into one modality by dual-use "theranostic" nanocarriers engineered to simultaneously deliver therapeutic and imaging cargoes [6, 7]. Imaging payloads, such as fluorescent, MRI, and radio tags can be loaded in the nanosystems or coated on their surface. Nanosized polymeric vesicles (polymersomes) selfassembled from biocompatible copolymers are particularly appealing because of their versatility and unique properties. The high molecular weight of block copolymers results in the formation of highly entangled membranes displaying a high degree of resilience with elastomer-like mechanical properties. This confers the polymersomes a higher flexibility $[8,9]$ and higher ability for tissue penetration than other vesicles self-assembled from low molecular weight entities, such as liposomes [10]. Polymersomes can be loaded with hydrophilic effector molecules, e.g. low molecular weight drugs [11,12], proteins [13], nucleic acids [14], and imaging agents [15, 16], in their aqueous lumen and with hydrophobic cargoes within the polymer membrane [11, 17].

The surface of nanoparticles can also be modified to improve their in vivo behavior such as circulation halflife, non-specific interactions and affinity for non-target sites, and to achieve selective accumulation in target tissue(s). Affinity ligands, such as peptides [18, 19] and antibodies [20] can be coated on the nanoparticles for specific tissue and cell recognition. Tumor-penetrating peptides [21] can be used to concentrate cytotoxic molecules and drug-loaded nanoparticles in tumors and potentiate their antitumor activity $[17,22]$. The AKRGARSTA peptide, referred to as "LinTT1" (linear TT1), is a 9-amino acid tumor-penetrating peptide that binds to $\mathrm{p} 32$ protein. The primary receptor for LinTT1, p32, is a mitochondrial chaperone that is aberrantly expressed at the cell surface of malignant cells and activated tumor macrophages, which makes it a good target molecule for affinity-based cancer delivery [23, 24]. A variety of solid tumors, such as glioblastoma and carcinomas of the gastrointestinal tract $[24,25,18]$, overexpress cell surface p32 and several studies have found upregulated $\mathrm{p} 32$ expression in TNBC [24, 26, 27]. LinTT1 is processed by tumor-derived proteases, such as uPA, to C-terminally expose the C-end rule motif of the peptide (i.e. AKRGAR), which is capable of interacting with the cell- and tissue-penetration receptor NRP-1 [23, 28] (Figure 1). Recently, LinTT1-functionalization was found to significantly improve the therapeutic index of iron oxide nanoworms loaded with proapoptotic effector peptide in a model of peritoneal carcinomatosis [18] and also in a TNBC model in mice [27]. In that last study, the tumor accumulation of fluorescently labeled LinTT1 nanoparticles was evaluated by optical imaging of tissue sections. However, fluorescence imaging-based in vivo biodistribution studies remain challenging due to issues related to the low depth of light penetration, tissue autofluorescence, and the semi-quantitative nature of optical imaging [29].

PET and SPECT are clinically used for imaging of radioactive contrast agents with beta and gamma emission, respectively. As the signal only comes from the radiotracer and as the tissues do not possess inherent radioactivity, PET and SPECT are not subject to endogenous tissue background, as opposed to MRI, CT, and imaging using optical contrast agents [30]. Moreover, in PET and SPECT the signal is not affected by tissue depth (as during in vivo imaging using fluorescent tags) or respiratory motion (as in the case in MRI) [30].

Encouraged by the anticancer activity of LinTT1targeted therapeutic nanoparticles on breast tumors in mice [27], we decided to evaluate polymersomes guided with the LinTT1 peptide as a potential theranostic nanocarrier to early detect TNBC lesions. (Figure 1) We radiolabeled LinTT1-targeted PEG-PCL polymersomes and studied, for the first time, the homing to orthotopic small breast tumors and the biodistribution of the polymersomes using PET imaging in mice. Intravenously-administered p32-targeted ${ }^{124} \mathrm{I}$ labeled polymersomes showed good tumor selectivity and, importantly, allowed detection of tumors smaller than $20 \mathrm{~mm}^{3}$. Our results suggest potential applications of LinTT1 engineered polymersomes for early detection of TNBC.

\section{RESULTS}

\section{Preparation, functionalization and radiolabeling of polymersomes}

Polymersomes were prepared by the film hydration method and functionalized with the Cys-Tyr-LinTT1 or Cys-FAM-LinTT1 peptide through a thioether bond between the maleimide group of the copolymer and the thiol group of the cysteine of the peptide. The number of polymersomes was determined by the ZetaView instrument 
and the functionalization with FAM-labeled peptide was quantified by fluorimetry. The peptide functionalization resulted in $\sim 3.7 \times 10^{4}$ peptides/polymersome particle (density $\sim 0.7$ peptides $/ \mathrm{nm}^{2}$ ). For radiolabeling, the polymersomes were functionalized with the Cys-Tyr-Ahx-LinTT1 (Ahx = aminohexanoic acid) peptide or control Cys-Tyr dipeptide. The tyrosine residue was incorporated for radioiodination. The hydrodynamic diameter of LinTT1-Tyr-polymersomes (LinTT1-Tyr-PS), Tyr-polymersomes (Tyr-PS), polymersomes labeled with ATTO550 (LinTT1-ATTO550PS and ATTO550-PS), and polymersomes labeled with FAM (LinTT1-FAM-PS and FAM-PS) measured by DLS, was $\sim 130 \mathrm{~nm}$ for all the polymersome samples (Figure 2B). As shown in Figure 2A, the size of polymersomes measured by TEM was smaller than 100nm. By TEM, we obtained the size of the dry polymersomes while DLS showed the hydrodynamic diameter of the particles in solution. In DLS the observed particle size was higher than in TEM due to DLS sample being in the solvated state with water molecules and ions associated with polymersomes [31]. Moreover, in the DLS, we used the intensity of the scattered light as a function of the particle size. As the intensity scales with the sixth power of the radius the larger particles have higher representation in the size distribution graphic. The Z-potential was slightly negative but very close to 0 for the different polymersome preparations (Figure 2B and Supplementary Figure 1). For the Z-potential measurements, we used a moderate ionic strength, $10 \mathrm{mM}$.
At high ionic strength, such as in phosphate saline buffer, the charges of the nanoparticles are screened by the ions in solution. At lower ionic strength, such as $10 \mathrm{mM} \mathrm{NaCl}$, this effect is lower and the zeta potential is closer to the potential resulting from the actual charge of particle [32]. Moreover, by using a known ionic strength, the influence of unknown concentration of ions contained in water was avoided.

For PET imaging, the LinTT1-Tyr-PS and TyrPS were radiolabeled with ${ }^{124} \mathrm{I}$. Before purification, the efficiency of polymersome radiolabeling was determined by TLC (thin layer chromatography) (Supplementary Figure 2). The yield of radiolabeling after purification, measured with activimeter, was $48 \pm 9 \%$ for LinTT1-Tyr-

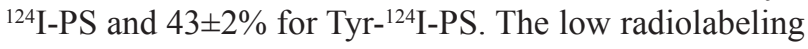
of PEG-PCL polymersomes without peptide $(14 \%$ or radiolabeling, Supplementary Figure 2) indicated that ${ }^{124} \mathrm{I}$ present in LinTT1-Tyr- ${ }^{124}$ I-PS and Tyr- ${ }^{124}$ I-PS preparations was predominantly due to the covalent binding of ${ }^{124} \mathrm{I}$ to the tyrosine residue of the peptides. TLC analysis after purification demonstrated that $99 \%$ of the ${ }^{124} \mathrm{I}$ was bound to polymersomes (Figure 2C).

\section{LinTT1-targeted polymersomes bind to recombinant $\mathrm{p} 32$ and to cultured breast tumor cells}

To evaluate the effect of LinTT1 functionalization on the tropism of polymersomes in vitro, we first tested the binding of LinTT1-Tyr- ${ }^{124}$ I-PS to human recombinant

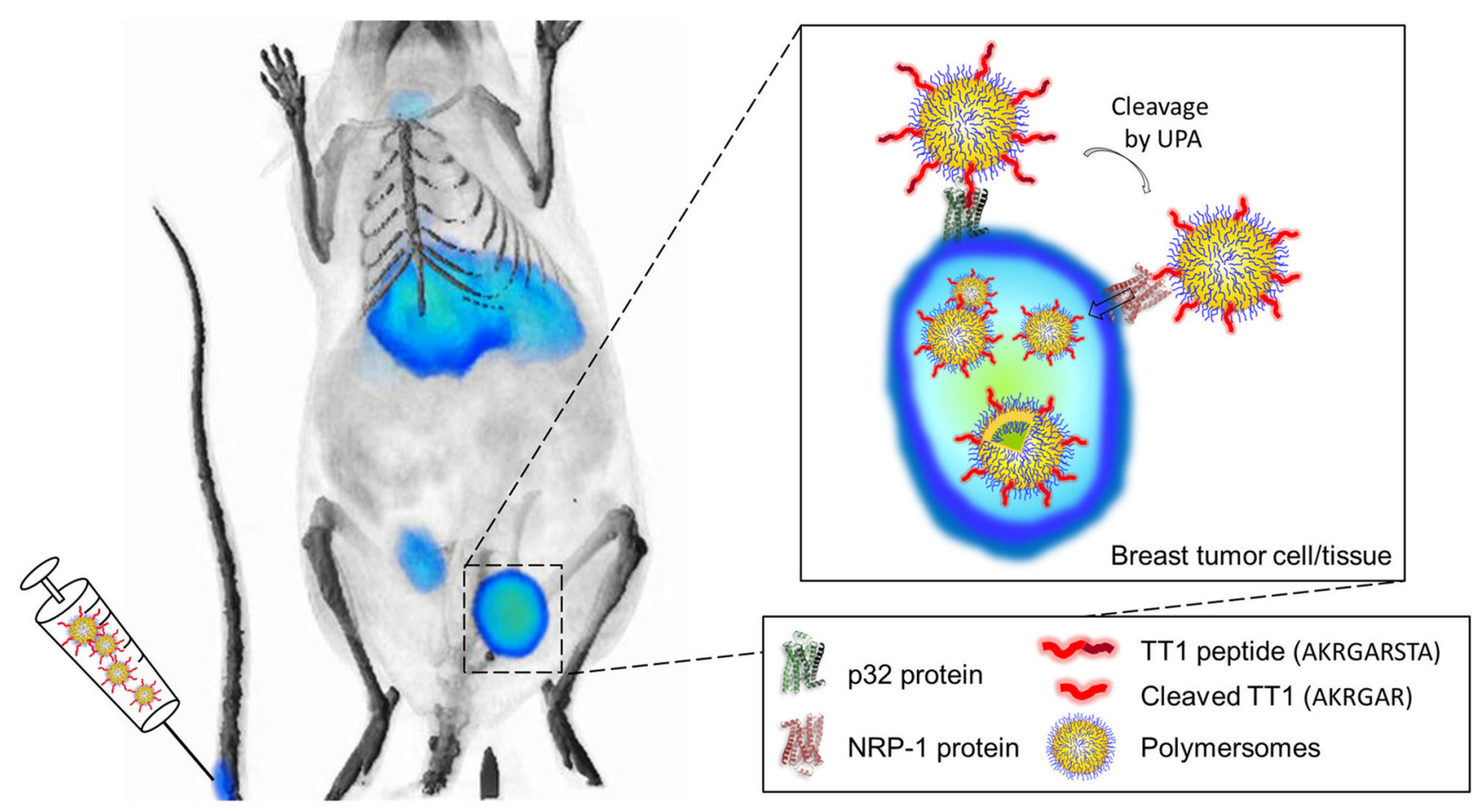

Figure 1: Small triple negative brast tumors can be detected with LinTT1-conjugated polymersomes. Radiolabeled LinTT1polymersomes were intravenously injected into mice bearing small triple negative breast tumor. LinTT1 peptide binds to p32 protein expressed in the surface of tumor cells and activated macrophage/myeloid cells. LinTT1 is cleaved by urokinase type plasminogen activator (uPA) in tumor, and the processed peptide binds to NRP-1, triggering the penetration of polymersomes into the tumor tissue. 
p32 protein, the primary receptor of LinTT1. P32-coated magnetic beads were incubated with the polymersomes, and polymersome binding was quantified by gamma counter. Compared to non-targeted polymersomes, LinTT1-Tyr- ${ }^{124}$ I-PS showed $\sim 10$-fold increased binding to the p32 beads (Figure 3A). This binding was specific, as the LinTT1-Tyr- ${ }^{124}$ I-PS did not bind to NRP-1 (Figure 3A). The LinTT1 peptide does not bind to NRP-1 unless proteolytically processed by uPA [28]. These data show that the LinTT1 peptide attached to the polymersomes remains available for human p32 binding to modulate polymersome tropism.

Various human and mouse tumor cell lines express p32 on the cell surface [24]. We studied the presence of cell surface p32 in 4T1 and MCF-10CA1a TNBC cells by flow cytometry and confocal microscopy, and confirmed its surface expression on both cell lines (Supplementary Figure 3). We tested the uptake of polymersomes and p32 co-localization in 4T1 cells - the same cell line used to establish the in vivo TNBC breast cancer model for systemic targeting studies. 4T1 cell line is of mouse origin and the tumors can be induced in immunocompetent Balb/c mice. The use of immunocompetent mice is an important aspect that is particularly relevant for the follow-up studies with LinTT1-guided combinations of companion diagnostic and therapeutic polymersomes. To study the uptake of polymersomes in 4T1 cells, we incubated the cultured cells for $1 \mathrm{~h}$ with LinTT1-targeted or control polymersomes labeled with ATTO550 (LinTT1ATTO550-PS and ATTO550-PS) (Figure 3B). The LinTT1-functionalization increased polymersome uptake in 4T1 cells and the signal from LinTT1-ATTO550-PS partially colocalized with p32 (Figure 3B). We also studied the uptake of LinTT1-PS in MCF10CA1a human breast tumor cultured cells. Supplementary Figure 4 showed a significantly higher uptake of LinTT1-PS compared with the non-targeted polymersomes. These experiments demonstrate that LinTT1 functionalization results in p32-enhanced uptake of polymersomes in cultured breast tumor cells.

\section{Systemic LinTT1 targeted radiolabeled polymersomes home to breast tumors}

We used PET imaging to study in vivo biodistribution and tumor accumulation of systemic LinTT1-targeted polymersomes. Radiolabeled LinTT1-Tyr- ${ }^{-124}$ I-PS and Tyr- ${ }^{124}$ I-PS were i.v. injected into mice bearing orthotopic $4 \mathrm{~T} 1$ breast tumors and PET-CT scans were acquired at 10 $\min , 2,6,12,24$, and $48 \mathrm{~h}$ post-injection. To test whether detection of incipient breast tumors could be improved by targeting p32, the polymersomes were administered when breast tumor had reached $\sim 20 \mathrm{~mm}^{3}$ (Figure 4).

LinTT1 functionalization increased tumor homing of polymersomes at both early and late time points (Figure 4C), with the AUC (area
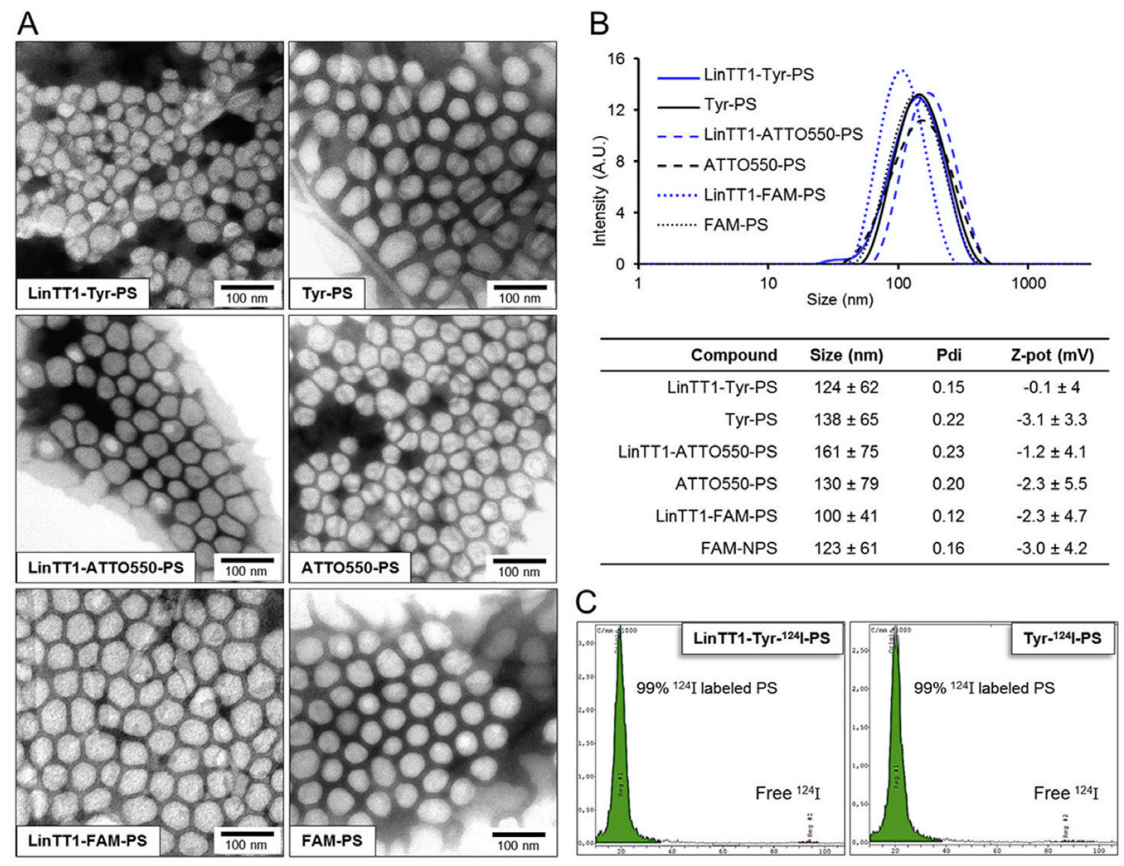

Figure 2: Characterization of the polymersomes. (A) TEM images of the LinTT1-targeted and non-targeted radiolabeled polymersomes (LinTT1-Tyr-124I-PS and Tyr-124I-PS) and fluorescently labeled polymersomes (LinTT1-ATTO550-PS, ATTO550-PS, LinTT1-FAM-PS, and FAM-PS). (B) Size distribution measured by DLS and summary of the physical properties of the polymersome preparations (3 independent measurements). (C) Chromatograms obtained by TLC of radiolabeled polymersomes after the purification showing the percentage of ${ }^{124} \mathrm{I}$-labeled polymersomes and the peak of free ${ }^{124} \mathrm{I}$. 
under the curve) in tumor being $\sim 60 \%$ higher (Figure 4D). We saw tumor accumulation of LinTT1Tyr- ${ }^{124} \mathrm{I}-\mathrm{PS}$ already at $2 \mathrm{~h}$ post injection, whereas the tumor PET signal for non-targeted Tyr- ${ }^{124} \mathrm{I}-\mathrm{PS}$ was only detectable at later time points (Figure 4A). The highest tumor accumulation of LinTT1-Tyr- ${ }^{124}$ I-PS was seen at $24 \mathrm{~h}$ after the injection, and it was $67 \%$ higher than for the untargeted polymersomes. At $48 \mathrm{~h}$ both targeted and untargeted polymersomes showed accumulation in the tumor (Figure 4A, 4B, 4E). At $48 \mathrm{~h}$ tumor accumulation of LinTT1-Tyr- ${ }^{124}$ I-PS was lower than at $24 \mathrm{~h}$, however, it was significantly higher than Tyr- ${ }^{124} \mathrm{I}-\mathrm{PS}(12 \pm 0.9$ and $9 \pm 0.4$ ID/cc, respectively) (Figure $4 \mathrm{C}$ ). In contrast, at $48 \mathrm{~h}$, the signal in the kidney and thyroid gland in mice injected with targeted and untargeted polymersomes was not significantly different (Figure 4E, Supplementary Figure 5).

The scans acquired at $6 \mathrm{~h}$ showed uptake of both targeted and non-targeted polymersomes in the liver (Figure 4A), in line with known role of the RES in the clearance of the circulating nanoparticles.

At $48 \mathrm{~h}$ after the injection, the tumors and organs were excised and ${ }^{124} \mathrm{I}$ in tissue extracts was quantified with gamma counter. The highest percentage of ID/g of both targeted and non-targeted polymersomes after $48 \mathrm{~h}$ was observed in spleen and tumor (Figure 5A). Accumulation of both LinTT1 and untargeted polymersomes in spleen is consistent with the polymersome clearance by the RES. At $48 \mathrm{~h}$, untargeted polymersomes showed accumulation in tumors $(15 \pm 0.6 \% \mathrm{ID} / \mathrm{g})$ and the functionalization with LinTT1 increased tumor accumulation of polymersomes by $>70 \%$, to $26 \pm 3 \% \mathrm{ID} / \mathrm{g}$. Moreover, the percentage of ID/g of LinTT1-Tyr- ${ }^{124}$ I-PS in tumor was 2.5 times higher than in liver (Figure 5A). Quantification of radioactivity revealed more than 2-fold higher accumulation of LinTT1Tyr- ${ }^{124} \mathrm{I}-\mathrm{PS}$ than Tyr- ${ }^{124} \mathrm{I}-\mathrm{PS}$ in the sentinel lymph node of breast tumor mice (Figure 5A).

The elimination rate of ${ }^{124} \mathrm{I}$ was studied by quantification of the PET imaging data. At $24 \mathrm{~h}, \sim 50 \%$ of the injected Tyr- ${ }^{124} \mathrm{I}-\mathrm{PS}$ and $67 \%$ of LinTT1-Tyr- ${ }^{124} \mathrm{I}-$ PS remained in the body. After $48 \mathrm{~h}, 32 \%$ of $\mathrm{Tyr}^{-124}{ }^{12} \mathrm{I}-\mathrm{IS}$ and $45 \%$ for LinTT1-Tyr- ${ }^{124}$ I-PS remained in the body (Figure 5B). We have shown in a recent publication that an insignificant portion of the peptide is released from PEG-PCL polymersomes incubated with the serum of the 4T1 tumor bearing mice for $6 \mathrm{~h}$ [33]. We suggest that the high excretion at short time points observed is due to the renal clearance of the ${ }^{124}$ I released from the peptide-conjugated polymersomes. It is important to note that the signal in thyroid gland (Supplementary Figure 5) - which accumulates free iodine - is similar for both targeted and untargeted polymersomes, suggesting similar leaching of iodine from polymersomes.

The effect of LinTT1 functionalization on the biodistribution and elimination rate of the polymersomes may be due to depletion of circulating LinTT1polymersomes by the target sites: tumor tissue and macrophages. 4T1 tumor mice injected with both LinTT1

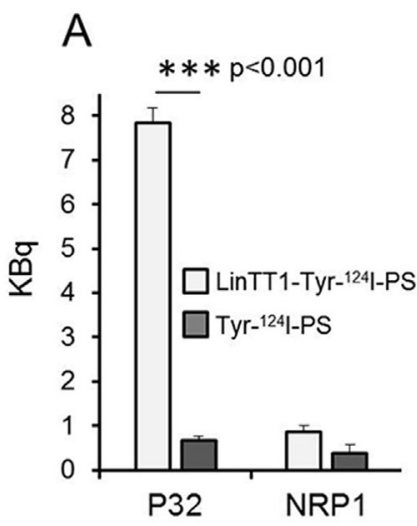

Recombinant protein
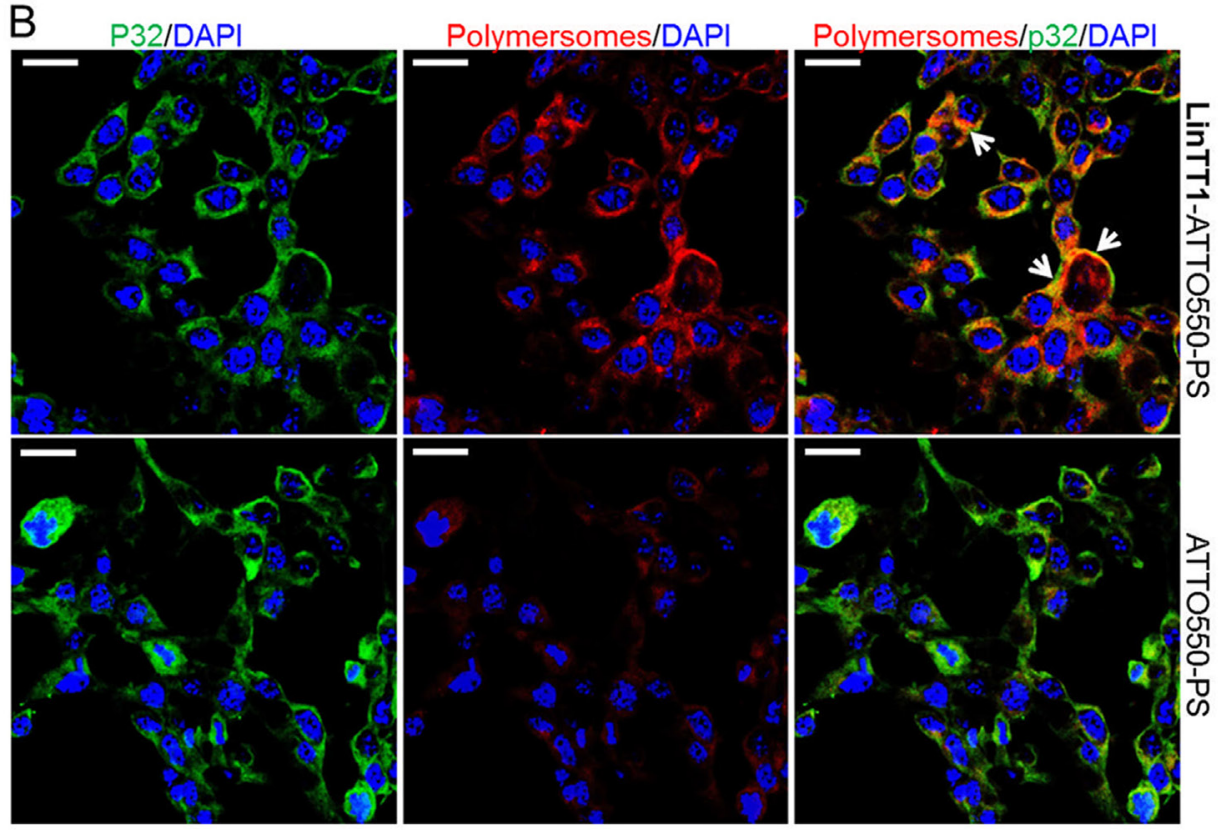

Figure 3: Binding of LinTT1-PS to recombinant p32 protein and to cultured 4T1 breast tumor cells. (A) Binding of the LinTT1-Tyr- ${ }^{124}$ I-PS and Tyr- ${ }^{124} \mathrm{I}-\mathrm{PS}$ to $\mathrm{p} 32$ and NRP1-coated magnetic beads. The binding to the proteins after $1 \mathrm{~h}$ of polymersome incubation is expressed in KBq. N=3. Error bar=+SEM. (B) Fluorescence confocal microscopy images of 4T1 cells incubated with LinTT1ATTO550-PS or non-targeted ATTO550-PS for $1 \mathrm{~h}$. The polymersomes were labeled with ATTO550 (red) and cells were immunostained for p32 protein (green). The nuclei were counterstained with DAPI (blue). Scale bar $=20 \mu \mathrm{m}$. White arrows point to the areas of colocalization of LinTT1-ATTO550-PS with p32. 
targeted and untargeted polymersomes showed similar ${ }^{124} \mathrm{I}$ excretion rate at short time points. However, after $6 \mathrm{~h}$, the ${ }^{124} \mathrm{I}$ excretion rates for the targeted polymersomes became lower, likely due to preferential uptake of LinTT1-Tyr- ${ }^{124} \mathrm{I}-$ PS by $\mathrm{p} 32^{+}$tumor cells and activated macrophages.

\section{LinTT1-polymersomes target both the tumor cells and tumor macrophages}

We next studied the tissue biodistribution of i.v. administered FAM-labeled polymersomes in 4T1 orthotopic tumor mice at the cellular level. The polymersomes were injected in 4T1 tumor mice, allowed to circulate for $24 \mathrm{~h}$, and the sections of tumors and control organs were analyzed by confocal immunoanalysis.

We first studied the biodistribution of p32 immunoreactivity in tissues. In a previous report, p32 was found to be upregulated in MDA-MB-435 breast tumors compared to the control organs [34, 24]. P32 immunostaining of sections of tumors and control organs from 4T1 mice demonstrated elevated expression of p32 in tumor tissue (Supplementary Figure 6).

In agreement with the tissue extract-based radiography data, FAM-LinTT1-polymersomes accumulated in tumor and spleen (Figure 4A). It was recently published that LinTT1 functionalization of
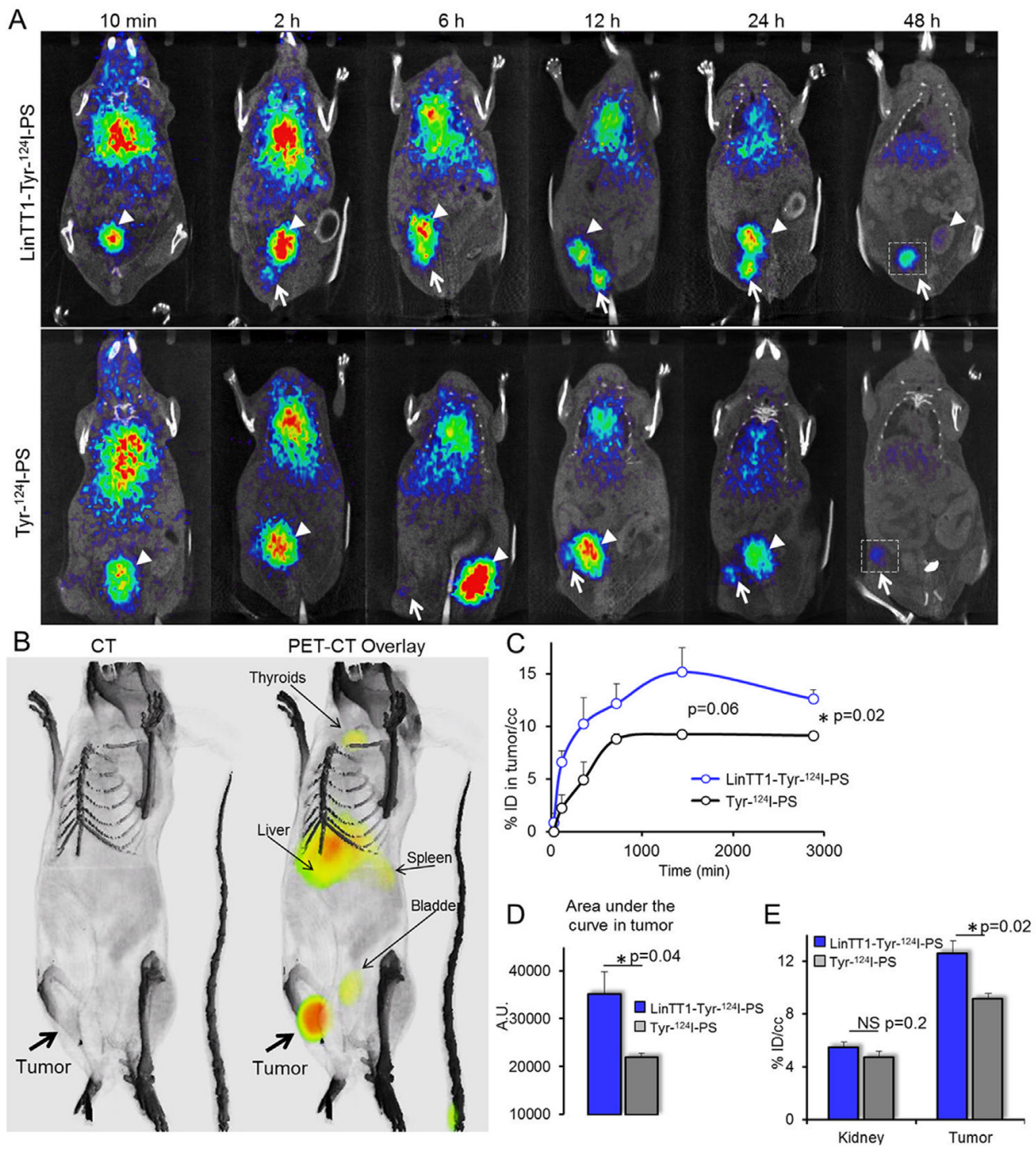

Figure 4: Radiolabeled LinTT1-PS home to 4T1 breast tumors. (A) PET-CT imaging of 4T1 tumor mice injected with LinTT1Tyr- ${ }^{124}$ I-PS or non-targeted Tyr- ${ }^{124}$ I-PS. White arrows point to the tumor. White arrowheads point to the bladder. The same mouse was used for the imaging at all the time points. The difference in tumor location is due to slightly different positioning of the mouse on the imaging support. (B) 3D reconstruction of CT and PET-CT overlay images of mouse at $48 \mathrm{~h}$ after LinTT1-Tyr- ${ }^{124} \mathrm{I}-\mathrm{PS}$ i.v. injection. (C) Accumulation of radiolabeled polymersomes in the tumor. The $\%$ of ID/cc tumor was plotted against the time post-injection. The signal was quantified from the PET images. (D) AUC of LinTT1-Tyr- ${ }^{124}$ I-PS and non-targeted Tyr- ${ }^{124}$ I-PS calculated the graph C. (E) \% ID/cc in kidney and tumor after $48 \mathrm{~h}$ of polymersome injection. The signal was quantified from the PET images. N=5 mice. Error bar=+SEM. 
nanoparticles enhances their penetration into tumor tissue $[27,18]$. Here we show that at $24 \mathrm{~h}$, the LinTT1-FAMPS in tumors did not colocalize with CD31-positive blood vessels, confirming that polymersomes had extravasated and penetrated into tumor stroma (Figure 5C, tumor inset). It has been shown that $\mathrm{p} 32$ is expressed by CD11b positive macrophages [34] and that LinTT1-conjugated nanoparticles colocalized with C68-positive macrophages in the breast [27], gastric, and colon tumors [18]. To study the macrophage uptake of LinTT1-PS, sections of tumors and organs were immunostained with antibodies against CD68, CD11b, and CD206 markers. CD68 and CD11b are pan-macrophage markers that label normal macrophages (including macrophages in spleen, lung, and in Kupffer cells in liver [33]), and TAMs [35]. CD206 is a marker of pro-tumor M2 macrophages [36], which promote tumor growth [37].

We found that LinTT1-FAM-PS colocalized with CD68 ( $>50 \%$ of colocalization), and showed partial colocalization with CD11b and CD206 (9\% and 21\% of colocalization, respectively) in tumors, confirming the targeting of tumor-associated macrophages (Figure 6A and 6B). CD68-positive macrophages, extensively found in sentinel lymph node and spleen, and in liver (to a lesser extent), also showed colocalization with LinTT1-FAM-PS (Supplementary Figure 7A and 7B), which might be one of the reasons for the higher accumulation of LinTT1-polymersomes in spleen and lymph node (Figure 5A) compared with untargeted polymersomes.
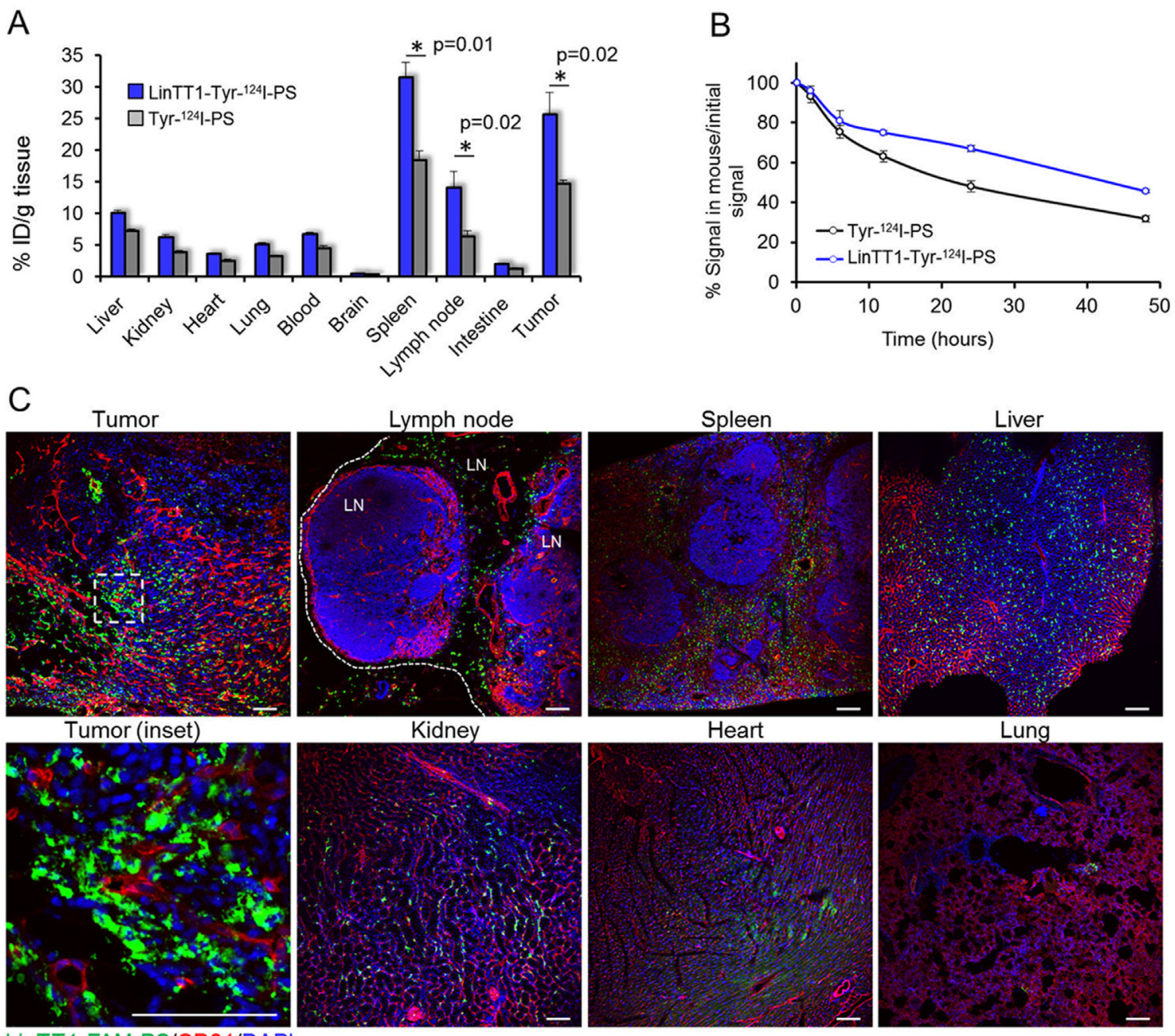

LinTT1-FAM-PS/CD31/DAP

Figure 5: Biodistribution of radioactive and fluorescent polymersomes in 4T1 tumor mice. (A) Biodistribution of i.v.injected ${ }^{124} \mathrm{I}$ labeled polymersomes in tumors and organs at $48 \mathrm{~h}$ after injection. Tumors, control organs, and blood were collected at $48 \mathrm{~h}$ post injection of radiolabeled polymersomes and the radioactivity was measured by gamma counter. $\mathrm{N}=6$. Error bar=+SEM. (B) Elimination rate of ${ }^{124} \mathrm{I}$ quantified from the PET data. The radioactive signal of the whole mouse was determined at different time points. $\mathrm{N}=5$ mice. Error bar=+SEM. (C) Confocal fluorescence imaging of sections of 4T1 tumors and control organs from mice injected i.v. with LinTT1-FAM-polymersomes. Tissues were collected at $24 \mathrm{~h}$ post-injection of polymersomes into $4 \mathrm{~T} 1$ bearing mice and sectioned and immunostained for FAM and CD31. Green: LinTT1-FAM-PS; red: CD31; blue: DAPI nuclear staining. LN= lymph node. 


\section{Human triple negative breast tumors and methastatic lymph nodes overexpress $\mathrm{p} 32$ and CD68+ macrophages}

To further investigate the clinical relevance and translatability of LinTT1-targeted polymersomes, we investigated the distribution of p32 and CD68 immunoreactivity on surgical cases of triple negative breast primary tumors and metastasis, in comparison to healthy breast tissue. As exemplified in Figure 7A and 7B, p32 diplays a uniform pattern of expression on healthy breast and healthy lymphoid tissue whereas strong membranous staining is found on malignant lesions. P32 is statistically significantly overexpressed in primary tumors, both metastatic and non-metastatic, and in particular on sentinel lymph nodes metastasis (Figure 7B, 7C). Additionally, increased number of CD68-positive cells was found in breast tumors and in sentinel lymph nodes, both from patients with and without metastases, in comparison to healthy breast (Figure 7D and Supplementary Figure 8). Partial colocalization of p32 and CD68 was also observed in malignant areas in metastatic lymph node (Figure 7B), demonstrating the expression of p32 in activated macrophages.

\section{DISCUSSION}

In the current study, we evaluated the LinTT1guided biocompatible PEG-PCL polymersomes as PET contrast agent for TNBC detection. Our findings indicate that LinTT1-polymersomes can be used for sensitive and specific detection of small triple negative breast tumors. This, along with recently published reports on LinTT1mediated targeting of therapeutic nanocarriers [18, 27], suggests potential theranostic applications for the LinTT1targeted nanocarriers for early detection and treatment of TNBC.

Nanoparticles have been affinity targeted to tumors for PET imaging. For example, in a recent PET study, clinical application of RGD-targeted PET-active nanoparticles for melanoma imaging has been reported [38]. Systemic nanoparticles of $\sim 100 \mathrm{~nm}$ are cleared by complement-mediated phagocytosis by Kupffer and endothelial cells of the liver as well as by phagocytic cells in the rest of the RES [39]. The polymersomes used in the current study are composed of biocompatible, biodegradable, and FDA approved PEG and PCL polymers [40]. In a dedicated toxicology study, the PEG-PCL-PEG micelles did not show signs of acute toxicity and there were no significant lesions found in histopathological study of major organs, including the liver and kidneys [41]. The maximum tolerated dose of these nanoparticles by i.v. administration was calculated to be 200 -fold higher than the dose used in our study.

The current study documents high tumor accumulation of LinTT1-polymersomes ( $>20 \%$ ID/ cc) that translates into an ability to detect very small malignant lesions, barely visible by CT (Supplementary Video 1). This sets our system apart from other molecular

A
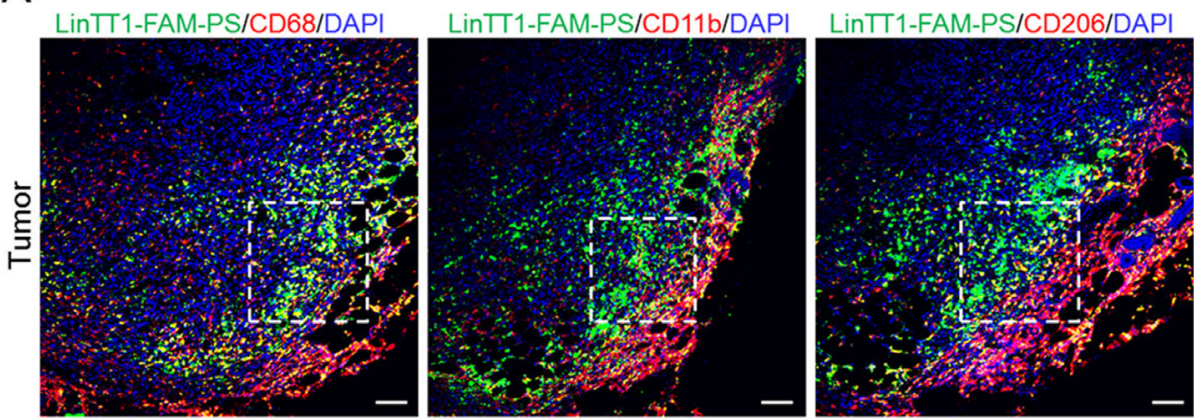

B
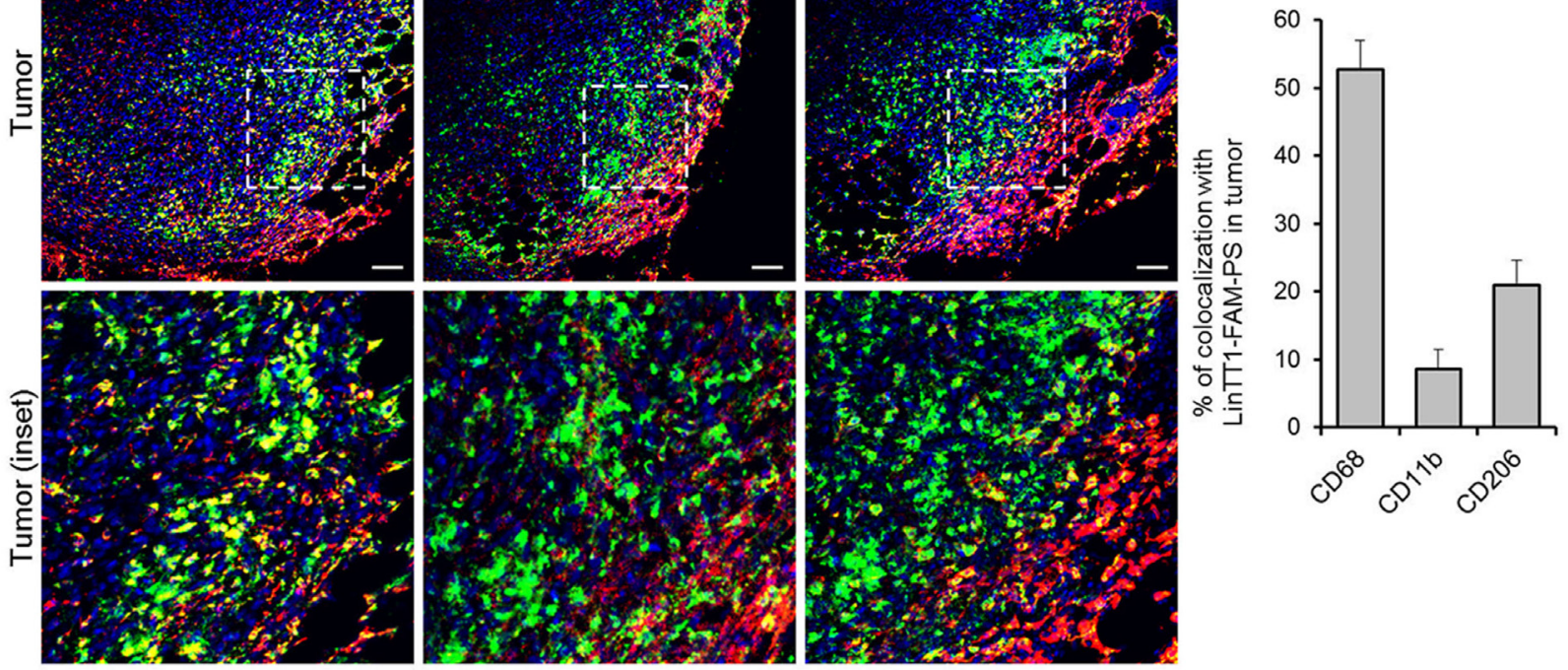

Figure 6: Colocalization of LinTT1-PS with macrophage markers in tumor tissue. $4 \mathrm{~T} 1$ tumors were collected at $24 \mathrm{~h}$ after LinTT1-FAM-PS i.v. injection into 4T1 bearing mice, sectioned and immunostained. (A) Confocal images of tumor sections immunostained for FAM, CD68, CD11b, and CD206, and counterstained with DAPI. Green: LinTT1-FAM-PS; red: CD68, CD11b, CD206; blue: DAPI counterstaining. Scale bar $=100 \mu \mathrm{m}$. (B) Quantification of the colocalization of LinTT1-FAM-PS and macrophage markers in tumor using FLUOVIEW Viewer software. Error bar=+SEM. 
and nanoparticle PET contrast agents with reported tumor accumulations between 5-10\% ID/g [42, 43, 44]. Remarkable tumor selectivity and tumor binding capacity observed for the LinTT1-polymersomes is likely to be due to a combination of the tumor homing properties of LinTT1 peptide with the favorable properties of the PEG-PCL polymersome nanoplatform. LinTT1 belongs to a family of tumor homing peptides that, unlike conventional vascular homing peptides, are not limited to vascular docking sites but have access to extended tumor extravascular space [45]. P32 protein, the receptor of LinTT1 peptide, is normally

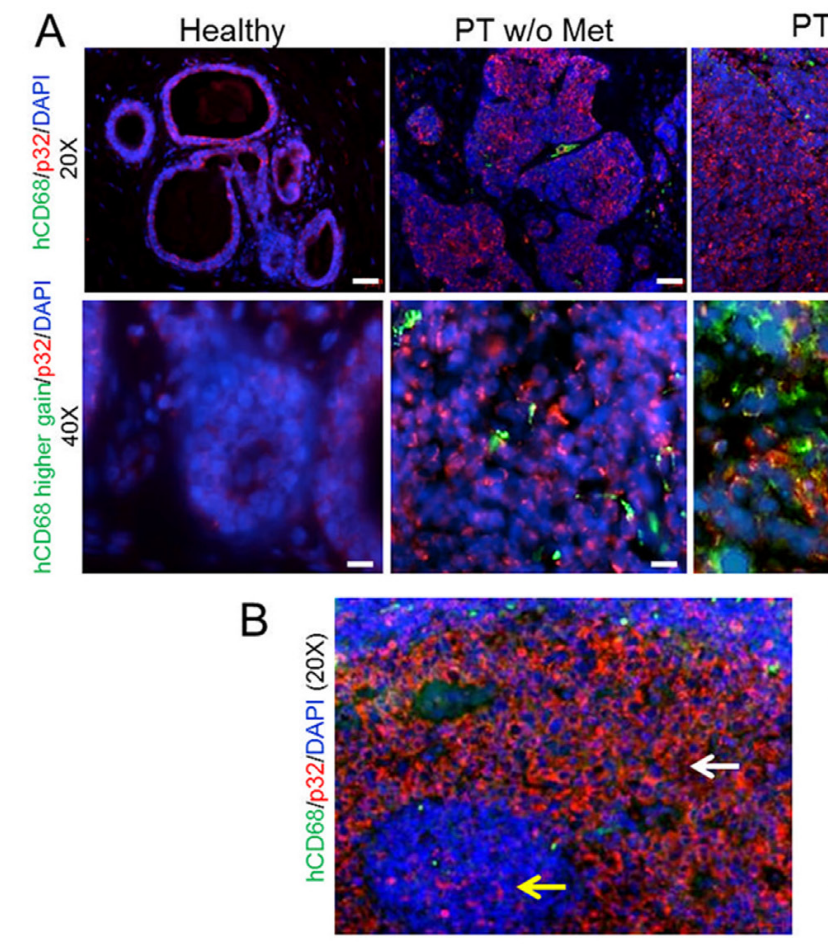

PT Met

LN w/o Met

LN Met
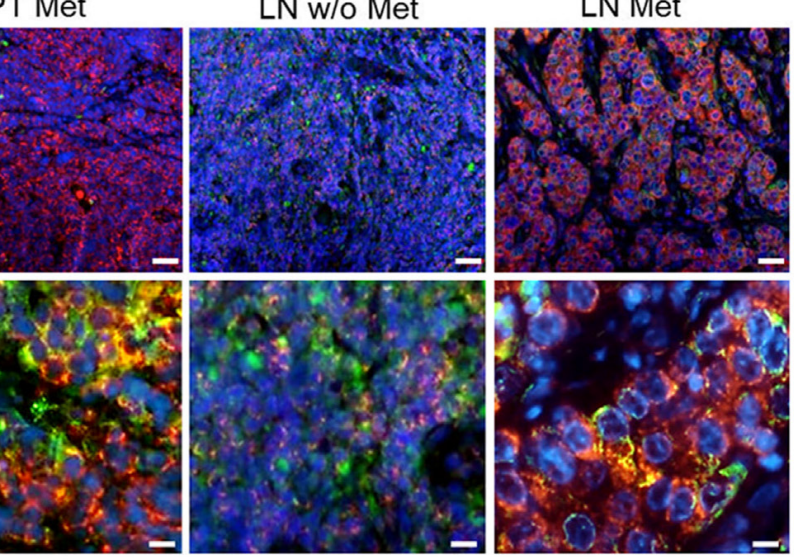

C
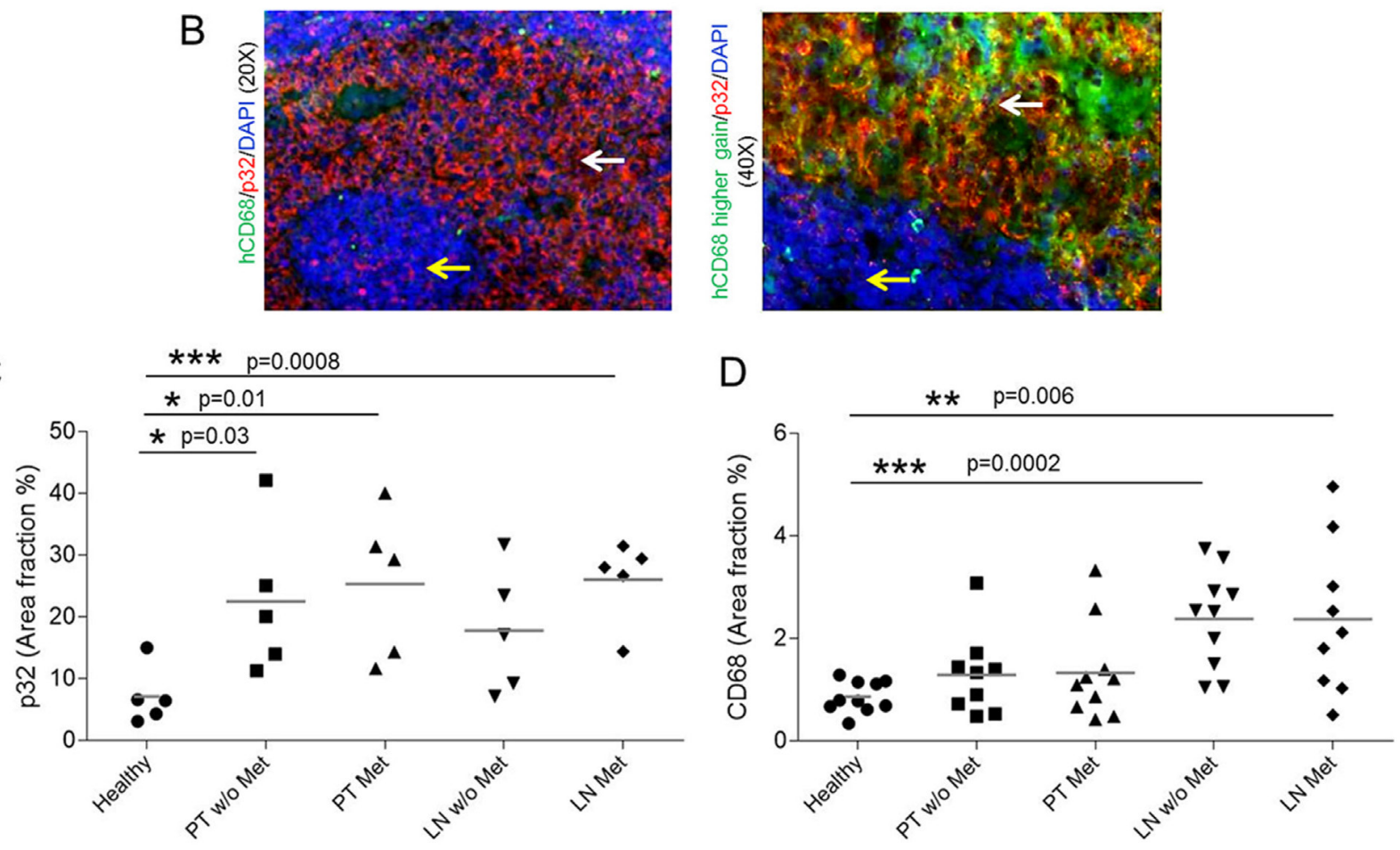

Figure 7: p32 and CD68 expression in human tissue samples. (A) Immunofluorescence-based staining of human p32 and CD68of FFPE human sections of healthy breast tissue (healthy), Primary Tumors with and without metastasis (PT Met and PT w/o Met, respectively) and their correspondent sentinel lymph nodes (LN Met and LN w/o Met, respectively), in N=5 patients/group. Representative pictures at 20x and 40x magnification. (B) Transitioning areas in metastatic lymph node showing immunostaining in healthy tissue (yellow arrows) and malignant lesions (white arrows) in lymph node. The green intensity was increased to show the colocalization of $\mathrm{p} 32$ and CD68. (C) Quantification of the p32 area fraction percentage in the different tissue sections. Three 20x representative images were acquired per patient and quantification was performed by the imaging-processing and analysis software AxioVision SE64 rel 4.8 .3 (Zeiss Microscopy). $\mathrm{N}=5$ patients/group. Scale bar for $20 \mathrm{x}=40 \mu \mathrm{m}$; scale bar for $40 \mathrm{x}=20 \mu \mathrm{m}$. (D) Quantification of the CD68 in the tissue sections from immunohistochemistry analysis. Four 20x representative images were acquired per patient and quantification was performed by the Automated Training Segmentation algorithm from the InForm Software (Perkin Elmer). N=10 patients/group. Scale bar for $4 x=400 \mu m$; scale bar for $20 \mathrm{x}=100 \mu \mathrm{m}$. 
expressed in the mitochondria of the cells, but it is aberrantly displayed on the surface of tumor cells and on macrophage/myeloid cells, specially in hypoxic areas of tumors $[23,24]$. LinTT1 binds to the superficial p32 on the tumor cells and activated macrophages and is cleaved by uPA, an enzyme involved in tumor migration and progression [46]. LinTT1 then exposes the CendR motif motive $(\mathrm{R} / \mathrm{KXXR} / \mathrm{K})$ on the $\mathrm{C}$-terminal. CendR motif binds to NRP-1 protein, which is overexpressed in tumor cells and tumor vasculature. The binding to NRP-1 triggers an increase of the tumor tissue permeability and the peptide together with the cargo is internalized into the tumor [47]. Another potentially contributing aspect, not addressed in the current study, is the ability of LinTT1 to increase tumor penetration of co-administered compounds and nanocarriers. LinTT1iron oxide nanoparticles were recently found to increase tumor penetration of co-administered $70 \mathrm{kDa}$ dextran [18]. Homing of LinTT1-nanocarriers may thus not be limited by the number of systemically accessible peptide receptors [48] and allow more nanocarriers to enter the tumor tissue for improved sensitivity of detection. Tumor accumulation of LinTT1-polymersomes may also be enhanced by physicochemical features of the PEG-PCL polymersomes used in the current study. On one hand, the flexibility of polymersomes [10] may contribute to tissue penetrative targeting with tumor-penetrating peptides. In addition, polymersomes are known to possess an intrinsic tumor tropism. The passive targeting of polymersomes presents an additional advantage for the tumor detection and treatment. We have recently demonstrated that $\mathrm{pH}$ sensitive polymersomes efficiently delivered payloads to the tumor tissue in the absence of active targeting [11]; this accumulation was further boosted by targeting with iRGD peptide [17]. Likewise, the systemic radiolabeled non-targeted polymersomes in the current study showed high accumulation in 4T1 breast tumors; this accumulation was potentiated by functionalization of polymersomes with the LinTT1 peptide by about $70 \%$. The effect of LinTT1 targeting is more prominent at earlier time points (at $2 \mathrm{~h}$ post-injection the LinTT1-PS, but not non-targeted PS, are already visible in the tumor, Figure 4), a useful feature that can reduce the imaging acquisition time for the tumor detection.

LinTT1 tumor homing is likely to be due to a combination of targeting of tumor cells and tumorassociated macrophages (TAMs). The targeting of both these cell populations increases the accumulation of LinTT1-PS in tumor to facilitate tumor detection. In the 4T1 breast tumor mice, the highest ID/g of LinTT1polymersomes was seen in the tumor, spleen, and lymph nodes. All these tissues contain abundant macrophages, a cell population known to upregulate, upon activation, the expression of cell surface p32. TAMs are an important therapeutic target, which play major roles in progression of solid tumors and can act as slow-release reservoir of drugs encapsulated in polymeric particles [49]. LinTT1polymersomes may be capable of targeting tumor cells and TAMs in TNBC patients, as the peptide is not species specific, and since TAMs are abundant in clinical lymph node and breast tumor samples. We show here that primary breast tumors and sentinel lymph nodes from clinical samples from patients overexpress p32 protein and that the number of CD68+ macrophages is increased compared with healthy tissues. This finding supports potential translatability of the system into clinical applications for the detection and treatment of TNBC. Clinical breast tumors are heterogeneous and the cell surface p32 expression and the sensitivity to p32-targeting-based treatment is likely to differ between the patients. PET imaging with LinTT1-polymersomes can be potentially used as a companion diagnostic test for selection of patient cohort most likely to respond to p32-targeted therapies.

Lymphangiogenesis in tumor-draining lymph nodes occurs before the onset of metastasis and is associated with distant metastasis. A study using Lyp-1 (a tumor lymphatics-specific peptide and also p32-binder [24]) to image tumor-induced lymphangiogenesis [50], suggested that the pre-malignant niche is positive for $\mathrm{p} 32$. The accumulation of LinTT1-polymersomes in sentinel lymph nodes containing 4T1 tumor cells migrating out from the primary tumor and activated macrophages, suggests potential applications for LinTT1-polymersomes for more precise detection of early metastatic dissemination of breast cancer than is possible with currently approved compounds, such as Lymphoseek [51].

The potential applications of our system extend beyond breast cancer detection and therapeutic targeting. Systemically accessible p32 is overexpressed across solid tumors, including, gastric, colon, and ovarian caricinoma [18], glioma (Säälik et al. unpublished), and in atherosclerosis lesions [52]. Systematic evaluation of the relevance of the linTT1-polymersomes for detection and/ or therapy of these conditions will be a subject of followup studies.

\section{MATERIALS AND METHODS}

\section{Materials}

Polyethylene glycol-polycaprolacone $\left(\mathrm{PEG}_{5000^{-}}\right.$ $\left.\mathrm{PCL}_{10000}, \mathrm{PEG}-\mathrm{PCL}\right)$ and Maleimide- $\mathrm{PEG}_{5000}-\mathrm{PCL}_{10000}$ (Mal-PEG-PCL) copolymers were purchased from Advanced Polymer Materials Inc. (Montreal, Canada). Cys-Tyr-Ahx-LinTT1 (Ahx = aminohexanoic acid) and Cys-Tyr peptides were purchased from KareBay Biochem, Inc. (USA), and Cys-fluorescein (FAM)TT1 and Cys-FAM peptides were purchased from TAG Copenhagen (Denmark). Sodium iodine-124 was purchased from Perkin Elmer (Amsterdam). Thin liquid chromatography sheets were purchased from Agilent Technologies (USA). The ATTO550-amine dye was 
purchased from Atto-Tech GmbH (Germany). 4T1 cells were purchased from (ATCC, CRL-2539) were cultured in RPMI medium 1640 + GlutaMAX with 25 mM HEPES (Gibco, Life Technologies, USA) containing $100 \mathrm{IU} / \mathrm{mL}$ of penicillin and streptomycin, and $10 \%$ of FBS (GE Healthcare, UK). MCF10CA1a cells were obtained from Erkki Rouslahti (Cancer Research Center, Sanford Burnham Prebys Medical Discovery Institute). The cells were maintained in Dulbecco's Modified Eagle Medium (DMEM, Lonza, Switzerland) with $4.5 \mathrm{mg} / \mathrm{mL}$ of glucose, $100 \mathrm{IU} / \mathrm{mL}$ of penicillin and streptomycin, and $10 \%$ of FBS.

\section{Synthesis and characterization of peptide-PEG- PCL vesicles}

PEG-PCL ( $8 \mathrm{mg}, 0.53 \mu \mathrm{mol})$ and Mal-PEGPCL ( $2 \mathrm{mg}, 0.13 \mu \mathrm{mol})$ copolymers were dissolved in $1 \mathrm{~mL}$ of acetone previously purged with nitrogen. The solvent was evaporated and the polymer film was hydrated with $1 \mathrm{~mL}$ of PBS $10 \mathrm{mM} \mathrm{pH} \mathrm{7.4,}$ previously purged with nitrogen. The suspension was sonicated for $5 \mathrm{~min}$ and the peptide (Cys-Tyr-LinTT1, Cys-Tyr, Cys-FAM-LinTT1peptide, or Cys-FAM, $0.4 \mathrm{mg}, 2 \mathrm{eq}$ ), dissolved in $0.2 \mathrm{~mL}$ PBS previously purged was added to the suspension. The suspension was sonicated for $30 \mathrm{~min}$, mixed at room temperature for $2 \mathrm{~h}$ and kept overnight at $4^{\circ} \mathrm{C}$. The vesicles were purified using centrifugal filters of $100 \mathrm{kDa} \mathrm{MWCO}$ (Amicon Ultra, Merck Millipore. Ltd. Ireland) and the final suspension was concentrated to $100 \mathrm{mg}$ of copolymer/mL.

For the polymersomes labeled with ATTO550, the dye was first conjugated to the polymer. Mal-PEGPCL $(10 \mathrm{mg}, 0.65 \mu \mathrm{mol})$ was dissolved in $0.3 \mathrm{~mL}$ of DMF previously purged with nitrogen and ATTO550$\mathrm{NH}_{2}(0.77 \mathrm{mg}, 2 \mathrm{eq})$ dissolved in $0.1 \mathrm{~mL}$ of previously purged DMF was added to the solution. Triethylene amine $(1 \mu \mathrm{L})$ was added to the solution as a catalyzer. The solution was reacted at room temperature overnight, dialyzed against water using dialysis membrane of $3.5 \mathrm{KDa} \mathrm{MWCO}$ (Sigma-Aldrich), and freeze-dried. ATTO550-PEG-PCL (1 mg, $0.06 \mu \mathrm{mol})$, PEG-PCL ( $7 \mathrm{mg}, 0.47 \mu \mathrm{mol}$ ) and Mal-PEG-PCL (2mg, $0.13 \mu \mathrm{mol})$ were dissolved in $0.5 \mathrm{~mL}$ of acetone. The solvent was then evaporated to form the polymer film. The polymersomes were assembled and the Cys-LinTT1 peptide conjugation was performed as described above.

DLS and Z-potential measurements (Zetasizer Nano ZS, Malvern Instruments, USA) were used to assess the average size, polydispersity, and surface charge of polymersome preparations. The size was measured at a concentration of $1 \mathrm{mg}$ polymer/mL in PBS $(10 \mathrm{mM}$ of phosphate and $137 \mathrm{mM}$ of $\mathrm{NaCl}$ ). The z-potential was measured at $0.2 \mathrm{mg}$ of polymer $/ \mathrm{mL}$ in $\mathrm{NaCl} 10$
$\mathrm{mM}$ ). TEM was used to assess the size and morphology of assembled vesicles. Briefly, polymersomes were deposited from a water solution onto copper grids at $1 \mathrm{mg} / \mathrm{mL}$, stained with $0.75 \%$ phosphotungstic acid $(\mathrm{pH}$ 7), air-dried, and imaged by TEM (Tecnai 10, Philips, Netherlands). The number of polymersomes in the suspension was measured using the ZetaWiew instrument (Particle Metrix GmbH, Germany).

\section{Iodination of PEG-PCL vesicles}

Two milligrams of Iodogen (Sigma-Aldrich, Spain) were dissolved in $10 \mathrm{~mL}$ of $\mathrm{CH}_{2} \mathrm{Cl}_{2}$ and $20 \mu \mathrm{L}$ of this solution was transferred to a tube and the solvent was evaporated. LinTT1-Tyr-polymersomes or Tyrpolymersomes $(1 \mathrm{mg})$ were mixed with $\mathrm{Na}^{124} \mathrm{I}(18.5 \mathrm{MBq})$ and $10 \mu \mathrm{L}$ of buffer phosphate $0.5 \mathrm{M}$ in a tube containing Iodogen. After $30 \mathrm{~min} 250 \mu \mathrm{L}$ of phosphate buffer, $1 \mathrm{M}$ $\mathrm{NaCl}, \mathrm{pH} 7.4$ was added to the reaction and the solution was transferred to a tube containing $50 \mu \mathrm{L}$ of $\mathrm{Na}_{2} \mathrm{~S}_{2} \mathrm{O}_{3} 0.1$ $\mathrm{M}$. The radiolabeling yield was measured by TLC using glass microfiber chromatography paper impregnated with silica gel (Agilent Technologies, USA) and ethanol:water $85: 15$ as mobile phase. The radioactivity of the peaks was measured with a TLC reader ( $\gamma$-MiniGITA, Raytest, Germany). The polymersomes were purified using centrifugal filters of $100 \mathrm{kDa}$ MWCO (Amicon Ultra, Merck Millipore. Ltd. Ireland) and resuspended in $0.1 \mathrm{~mL}$ of PBS. The removal of the free ${ }^{124} \mathrm{I}$ was confirmed by radio-TCL and the final radioactivity was measured with a dose calibrator (Capintec CRC-25R, USA).

\section{In vitro binding of polymersomes to recombinant p32 protein}

Recombinant hexahistidine-tagged p32 was bacterially expressed and purified as previously described [23]. For protein binding assays, Ni-NTA magnetic agarose beads (Qiagen, Germany) in binding buffer ( 50 $\mathrm{mM}$ Tris $\mathrm{pH} 7.4,150 \mathrm{mM} \mathrm{NaCl}, 5 \mathrm{mM}$ imidazole) were coated with p32 protein at $15 \mu \mathrm{g}$ of protein $/ 10 \mu \mathrm{L}$ beads. Radiolabeled polymersomes were incubated with the p32-coated beads in binding buffer containing 1\% BSA at room temperature for $1 \mathrm{~h}$. The magnetic beads were washed with binding buffer and resuspended in a final volume of $1 \mathrm{~mL}$ of binding buffer. The radioactivity of each sample was quantified by automatic gamma counter (2470 Wizard 2, Perkin Elmer).

\section{Assessment of in vitro cell surface $\mathrm{p} 32$ expression by flow cytometry}

4T1 and MCF10CA1a cells were detached from the culture plate with enzyme-free cell dissociation buffer (Gibco, Life Technologies, UK). The cells ( $10^{5}$ cells) were incubated with $10 \mu \mathrm{g} / \mathrm{mL}$ of the in-house generated rabbit 
polyclonal p32 antibody in blocking buffer containing $1 \%$ of BSA, $1 \% \mathrm{FBS}$, and $1 \%$ of goat serum in PBS at room temperature for $1 \mathrm{~h}$. The cells were washed, and incubated with Alexa 647-conjugated goat anti-rat IgG (1/1000, Invitrogen, Thermo Fisher Scientific, MA, USA) in blocking buffer at room temperature for $30 \mathrm{~min}$. After washes, the cell surface p32 expression was analyzed by flow cytometry (Accuri, BD Biosciences, CA, USA).

\section{Assessment of in vitro cell surface $\mathrm{p} 32$ expression by immunostaining}

4T1 and MCF10CA1a cells $\left(10^{4}\right)$ were seeded on glass coverslip in a 24 well-plate and incubated overnight at $37^{\circ} \mathrm{C}$. The cells were blocked in PBS containing $0.05 \%$ Tween-20, 5\% FBS, 5\% BSA, and 5\% goat serum (GE Healthcare, UK) for $1 \mathrm{~h}$. The cells were immunostained with $10 \mu \mathrm{g} / \mathrm{mL}$ of the in-house generated rabbit polyclonal p32 antibody in buffer containing $1 \%$ of BSA, $1 \%$ FBS, and $1 \%$ of goat serum in PBS at room temperature for $1 \mathrm{~h}$. The cells were washed, and incubated with Alexa 647-conjugated goat anti-rat IgG (1/2000, Invitrogen, Thermo Fisher Scientific, MA, USA) in blocking buffer at room temperature for $30 \mathrm{~min}$. Cells were counterstained with $1 \mu \mathrm{g} / \mathrm{mL}$ of DAPI, transferred to glass slides, and examined by fluorescence confocal microscopy using the Zeiss LSM710 instrument.

\section{Uptake of polymersomes in cultured cells}

$4 \mathrm{~T} 1$ cells $\left(5 \times 10^{5}\right)$ were seeded on glass coverslips in a 24-well plate and the next day incubated with ATTO550labeled polymersomes $(0.5 \mathrm{mg}$ polymer $/ \mathrm{mL})$ at $37^{\circ} \mathrm{C}$ for $1 \mathrm{~h}$. Cells were washed with PBS, fixed with $4 \%$ paraformaldehyde, permeabilized with $0.5 \%$ saponin, and blocked for $1 \mathrm{~h}$ with $1 \%$ BSA, $1 \%$ goat serum, $0.3 \mathrm{M}$ of glycine, and $0.05 \%$ of Tween-20 in PBS. Cells were then stained for p32 protein using anti-p32 rabbit polyclonal antibody (1/500, Millipore, Germany) and Alexa Fluor 488 goat anti-rabbit $\operatorname{IgG}(1 / 1000$, Abcam, UK) as a secondary antibody, and counterstained with $1 \mu \mathrm{g} / \mathrm{mL}$ of DAPI. Cells were examined under the confocal laser scanning microscope (LSM 510 Meta, Zeiss, Germany) equipped with a $63 \mathrm{x}$ oil objective lens (1.4 NA). Images were acquired sequentially to avoid cross-talk using excitation wavelengths 405, 488, and $561 \mathrm{~nm}$. Transmission images were collected and overlaid by Zeiss Zen software.

\section{In vivo PET-CT imaging}

For tumor induction, 1 million $4 \mathrm{~T} 1$ cells in $50 \mu \mathrm{L}$ of PBS were orthotopically implanted in the mammary gland of Balb/c mice (Charles River Laboratories, Spain). After 3 days, when the tumor volume had reached $\sim 18 \mathrm{~mm}^{3}$, the mice were injected in tail vein with $3.7-7.4 \mathrm{MBq}$ of radiolabeled polymersomes ( $1 \mathrm{mg}$ polymer, $100 \mu \mathrm{L}, \mathrm{N}=5$ mice) and subjected to PET-CT scans. During the scan acquisitions the mice were kept anesthetized with 1.5-2\% isofluorane blended with $\mathrm{O}_{2}$. The PET-CT scans were performed at $10 \mathrm{~min}, 2,6,12,24$, and $48 \mathrm{~h}$ using the Argus PET-CT scanner (Sedecal, Molecular Imaging, Spain). First, PET scans were acquired using the following acquisition protocol: whole body emission scan, 2 beds, 10 min of total acquisition time for the scans at $10 \mathrm{~min}, 2,6,12$, and 24 $\mathrm{h}$ post-injection and $20 \mathrm{~min}$ for the $48 \mathrm{~h}$ time point. The acquisition method was static, using $400-700 \mathrm{KeV}$ energetic window, FBP reconstruction algorithm, with correction for scatter coincidences. For the CT scans the used current was $140 \mu \mathrm{A}, 40 \mathrm{kV}$ of voltage, rotation of 360 degrees, 4 shoots, 1 bed, acquisition time of $6 \mathrm{~min}$, and a reconstruction binning of 2 . After $48 \mathrm{~h}$ of radiolabeled polymersome injection the mice were sacrificed and the tumor, blood, and organs were excised and further used for biodistribution studies. The PET-CT images were processed with the Medical Image Data Examiner AMIDE software. The CT and PET images were overlaid, the tumor volume was manually extracted from the CT scans and the same ROI was applied in the PET images, averaged, and expressed as percentage of injected dose per cubic centimeter of tissue (ID/cc). For the rendered 3D PET-CT images, PMOD image analysis software (PMOD Technologies Ltd, Zürich, Switzerland) was used. A 3D Gauss Filter of $1.5 \times 1.5 \times 1.5 \mathrm{~mm}$ was applied to the PET image in order to increase the signal to noise ratio for $3 \mathrm{D}$ visualization.

\section{Biodistribution studies}

For biodistribution studies, the tumor, blood, and organs were weighed and the radioactivity was measured using the automatic gamma counter. A standard curve was generated using ${ }^{124} \mathrm{I}$ to determine the relationship between cpm and Bq. The biodistribution was expressed as percentage of injected dose per gram of tissue (ID/g). To determine the elimination rate of polymersomes, the radioactive signal in the whole mouse body was measured from the PET images at $10 \mathrm{~min}, 2,6,12,24$, and $48 \mathrm{~h}$ post-injection and normalized by the signal at 10 min postinjection. The elimination rate was expressed as signal in mouse x 100 divided by the signal at time zero.

\section{Tissue immunofluorescence and confocal microscopy}

Balb/c mice were orthotopically injected with 1 million of 4T1 cells in the mammary gland and after 3 days FAM-LinTT1-PS (1mg of polymer, $100 \mu \mathrm{L}$ ) was intravenously injected. After $24 \mathrm{~h}$, the animals were sacrificed and the tumor and organs were excised, fixed in $4 \%$ of paraformaldehyde, cryoprotected with $15 \%$ and $30 \%$ sucrose, frozen down with liquid nitrogen, and cryosectioned at $10 \mu \mathrm{m}$. Tissue sections were permeabilized using PBS $10 \mathrm{mM}$ containing $0.2 \%$ Triton- $\mathrm{X}$ for $10 \mathrm{~min}$, and blocked in PBS 10mM containing 0.05\% 
Tween-20, 5\% FBS, 5\% BSA, and 5\% goat serum (GE Healthcare, UK) for $1 \mathrm{~h}$. The sections were immunostained at dilution 1/100 with anti-fluorescein rabbit IgG fraction (Thermo Fisher Scientific, MA, USA), rat anti-mouse CD31, biotin rat anti-mouse CD11b, (BD Biosciences, CA, USA), rat anti-mouse CD68, rat anti-mouse CD206 (Bio-Rad, USA), and anti-p32 rabbit polyclonal antibody (Millipore, Germany) as primary antibodies. As secondary antibodies, Alexa 488-conjugated goat anti-rabbit IgG and Alexa 647-conjugated goat anti-rat IgG (1/500, Invitrogen, Thermo Fisher Scientific, MA, USA) were used. The sections were counterstained with DAPI and examined by fluorescence confocal microscopy using Olympus FV1200MPE instrument. The images were processed and analyzed using the FV10-ASW 4.2 Viewer image software (Olympus, Germany) and the Image J software.

\section{Immunofluorescence staining and quantification of p32 and CD68 in human tissue of breast tumor, lymph node, and healthy tissue}

Surgical samples of TNBC with lymph node metastasis (PT (Primary Tumor) Met; $n=5$ ), TNBC without lymph node metastasis (PT w/o Met; $n=5$ ) as well as their corresponding metastasized sentinel lymph nodes (LN Met; $n=5$ ) and non-metastasized lymph nodes (LN w/o Met; $n=5$ ) and healthy breast tissue (healthy; $n=5)$, were fixed for 12-24 hours in 4\% neutral buffered formalin. Samples were dehydrated, embedded in paraffin, sectioned at $4 \mu \mathrm{m}$, and mounted on coated microscope slides (Dako, Denmark). After deparaffinization and rehydration of the sections, antigen retrieval was performed in citrate buffer at $\mathrm{pH} 6$ followed by endogenous peroxidase blocking for $10 \mathrm{~min}$. The samples were then incubated for $1 \mathrm{~h}$ with blocking solution containing 5\% FBS, 5\% BSA, 5\% donkey serum, and 5\% goat serum in PBS, followed by incubation with rabbit anti-p32 antibody (1:200, made in house) and mouse anti-human CD68 antibody (1:200, Dako) at room temperature for $2 \mathrm{~h}$. Next, slides were incubated at room temperature for $1 \mathrm{~h}$ with the secondary antibodies Cy3-conjugated donkey anti-rabbit IgG (1:500, Dianova) and Alexa 488-conjugated goat anti-mouse IgG (1:50, Dianova). The sections were counterstained with DAPI and examined by fluorescence microscopy (Zeiss ${ }^{\circledR}$ AxioImager M2 microscope). The quantification was performed by the imaging-processing and analysis software AxioVision SE64 rel 4.8.3. All stainings were performed on archived FFPE human samples and approved by the local ethics committee (EK 039/17).

\section{Immunohistochemistry staining and quantification of CD68 in human tissue of breast tumor, lymph node, and healthy tissue}

Surgical samples of TNBC with lymph node metastasis (PT Met; $\mathrm{n}=10$ ), TNBC without lymph node metastasis (PT w/o Met; $\mathrm{n}=10$ ) as well as their corresponding metastasized sentinel lymph nodes (LN Met; $\mathrm{n}=10$ ) and non-metastasized lymph nodes (LN w/o Met; $n=10$ ) and healthy breast tissue (healthy; $n$ $=10$ ), were fixed for 12-24 hours in 4\% neutral buffered formalin. In two cases with neoadjuvant chemotherapy and in two cases where only very small residual tumor was found in the surgical specimen, specimens from corresponding diagnostic biopsies were selected for immunohistochemistry. Samples were dehydrated, embedded in paraffin, sectioned at $4 \mu \mathrm{m}$, and mounted on coated microscope slides (Dako, Denmark). After deparaffinization and rehydration of the sections, antigen retrieval was performed in citrate buffer at $\mathrm{pH} 6$ in a pre-treatment module (PT-Link, Dako, Denmark). Using an autostainer (Thermo Fisher Scientific) slides were incubated with endogenous peroxidase blocking solution for $5 \mathrm{~min}$, followed by mouse anti-human CD68 antibody (1/100, Dako) for $30 \mathrm{~min}$. Next, slides were incubated with secondary antibodies goat anti-mouse IgG conjugated to a peroxidase-labeled polymer chain (Dako, Denmark) for $20 \mathrm{~min}$. The antigen-antibody-polymer complex was visualized with DAB + Chromogen (Dako, Denmark) for $10 \mathrm{~min}$. The counterstaining was performed with Hematoxylin (Dako, Denmark) for 5 min. Finally, slides were covered with coverslipping film (Sakura, 6132 Prisma $\left.{ }^{\circledR}\right)$ and examinated with Vectra ${ }^{\circledR} 3$ automated quantitative pathology imaging system (Perkin Elmer). Quantification was performed by the Automated Training Segmentation algorithm from the InForm Software (Perkin Elmer). All stainings were performed on archived FFPE human samples and approved by the local ethics committee (EK 039/17).

\section{Statistical analysis}

All the statistical analysis was performed with the Statistica 8 software, using the one-way ANOVA, Fisher LSD test.

\section{Abbreviations}

TNBC: triple negative breast cancer; PEG-PCL: polyethylene glycol-polycaprolactone; ID: injected dose; cc: cubic centimeter; MRI: magnetic resonance imaging; uPA: urokinase type plasminogen activator; NRP1: neuropilin-1; PET: positron emission tomography; SPECT: single photon emission computed tomography; CT: computed tomography; FAM: fluorescein; DLS: dynamic light scattering; TLC: thin layer chromatography; i.v.: intravenously; AUC: area under the curve; RES: reticuloendothelial system; TAMs: tumor-associated macrophages; FFPE: formalin-fixed paraffin-embedded; FBS: fetal bovine serum; PBS: phosphate buffer saline; DMF: dimethyl formamide; TEM: transmission electron microscopy; BSA: bovine serum albumin. 


\section{Author contributions}

Lorena Simón-Gracia: Study conception, experiment design, polymersome synthesis, radiolabeling, PET-CT scan acquisition, flow cytometry, cells and tissue immunostaining, microscope pictures acquisition, data analysis, manuscript writing.

Pablo Scodeller: Study conception, experiment design, PET-CT scan acquisition, PET images analysis and quantification, cell immunostaining, microscope pictures acquisition, breast tumor induction, manuscript writing and revision

Sergio Salazar Fuentes: Performance of animal experiments: breast tumor induction, animal manipulation during the PET-CT scans, tumor and organs excision

Vanessa Gómez Vallejo: supervision of the radiolabeling

Xabier Ríos: practical support for the radiolabeling

Eneko San Sebastián: practical support for the PET-CT scans, PET-CT images analysis

Valeria Sidorenko: polymersome uptake in cultured cells

Desirè Di Silvio: practical support for the confocal microscope pictures acquisition

Meina Suck, Federica De Lorenzi, Larissa Yokota Rizzo: Immunostaining of the clinical samples, microscope pictures acquisitions, and data analysis

Saskia von Stillfried: Analysis and interpretation of the immunohistochemistry of clinical samples

Kalle Kilk: polymersome characterization

Twan Lammers: Critical revision of the manuscript

Sergio E Moya: Manuscript discussion, edition, and critical revision

Tambet Teesalu: Manuscript writing, discussion, edition, and critical revision

\section{ACKNOWLEDGMENTS}

We acknowledge Prof. Erkki Ruoslahti (Cancer Research Center, Sanford Burnham Prebys Medical Discovery Institute) for critical reading of the manuscript. Rein Laiverik (Department of Anatomy, University of Tartu) and Angel Martinez Villacorta (CIC Biomagune) are acknowledged for their help with the TEM equipment in this work.

\section{CONFLICTS OF INTEREST}

The authors have no conflicts of interest.

\section{FUNDING}

This work was supported by the European Regional Development Fund Mobilitas Plus postdoctoral fellowship MOBJD11 (to L. Simon-Gracia), the European Union through the H2020 PEOPLE RISE project HYMADE (645686) and the European Regional
Development Fund (Project No. 2014-2020.4.01.150012), by EMBO Installation grant \#2344 (to $T$. Teesalu), European Research Council starting grant GLIOMADDS from European Regional Development Fund (to T. Teesalu), and Wellcome Trust International Fellowship WT095077MA (to T. Teesalu). This project was also financially supported by the European Union (EU-EFRE: European Fund for Regional Development: I3-STM 0800387), by the European Research Council (ERC-StG-309495:NeoNaNo) and by the German Research Foundation (LA 2937/1-2 and SFB 1066). D. Di Silvio, and S. Moya acknowledge the ERA-NET SIINN FATENANO for support.

\section{REFERENCES}

1. Ovcaricek T, Frkovic SG, Matos E, Mozina B, Borstnar $\mathrm{S}$. Triple negative breast cancer - prognostic factors and survival. Radiol Oncol. 2011; 45:46-52. https://doi. org/10.2478/v10019-010-0054-4.

2. Lehmann BD, Bauer JA, Chen X, Sanders ME, Chakravarthy AB, Shyr Y, Pietenpol JA. Identification of human triple-negative breast cancer subtypes and preclinical models for selection of targeted therapies. J Clin Invest. 2011; 121:2750-67. https://doi.org/10.1172/ JCI45014.

3. Lehmann BD, Jovanović B, Chen X, Estrada MV, Johnson KN, Shyr Y, Moses HL, Sanders ME, Pietenpol JA. Refinement of triple-negative breast cancer molecular subtypes: implications for neoadjuvant chemotherapy selection. PLoS One. 2016; 11:e0157368. https://doi. org/10.1371/journal.pone.0157368.

4. Dent R, Trudeau M, Pritchard KI, Hanna WM, Kahn HK, Sawka CA, Lickley LA, Rawlinson E, Sun P, Narod SA. Triple-negative breast cancer: clinical features and patterns of recurrence. Clin Cancer Res. 2007; 13:4429-34. https:// doi.org/10.1158/1078-0432.CCR-06-3045.

5. Mann AP, Scodeller P, Hussain S, Joo J, Kwon E, Braun GB, Mölder T, She ZG, Kotamraju VR, Ranscht B, Krajewski S, Teesalu T, Bhatia S, et al. A peptide for targeted, systemic delivery of imaging and therapeutic compounds into acute brain injuries. Nat Commun. 2016; 7:11980. https://doi. org/10.1038/ncomms 11980 .

6. Lammers T, Aime S, Hennink WE, Storm G, Kiessling F. Theranostic nanomedicine. Acc Chem Res. 2011; 44:1029 38. https://doi.org/10.1021/ar200019c.

7. Kunjachan S, Ehling J, Storm G, Kiessling F, Lammers T. Noninvasive imaging of nanomedicines and nanotheranostics: principles, progress, and prospects. Chem Rev. 2015; 115:10907-37. https://doi.org/10.1021/ cr500314d.

8. Discher BM, Won YY, Ege DS, Lee JC, Bates FS, Discher DE, Hammer DA. Polymersomes: tough vesicles made from diblock copolymers. Science. 1999; 284:1143-46. https://doi.org/10.1126/science.284.5417.1143. 
9. Bermudez H, Brannan AK, Hammer DA, Bates FS, Discher DE. Molecular weight dependence of polymersome membrane structure, elasticity, and stability. Macromolecules. 2002; 35:8203-08. https://doi. org/10.1021/ma0206691.

10. Pegoraro C, Cecchin D, Madsen J, Warren N, Armes SP, MacNeil S, Lewis A, Battaglia G. Translocation of flexible polymersomes across pores at the nanoscale. Biomater Sci. 2014; 2:680-92. https://doi.org/10.1039/C3BM60294J.

11. Simón-Gracia L, Hunt H, Scodeller PD, Gaitzsch J, Braun GB, Willmore AM, Ruoslahti E, Battaglia G, Teesalu T. Paclitaxel-loaded polymersomes for enhanced intraperitoneal chemotherapy. Mol Cancer Ther. 2016; 15:670-79. https://doi.org/10.1158/1535-7163. MCT-15-0713-T.

12. Pegoraro C, Cecchin D, Gracia LS, Warren N, Madsen J, Armes SP, Lewis A, Macneil S, Battaglia G. Enhanced drug delivery to melanoma cells using PMPC-PDPA polymersomes. Cancer Lett. 2013; 334:328-37. https://doi. org/10.1016/j.canlet.2013.02.007.

13. Wang L, Chierico L, Little D, Patikarnmonthon N, Yang Z, Azzouz M, Madsen J, Armes SP, Battaglia G. Encapsulation of biomacromolecules within polymersomes by electroporation. Angew Chem Int Ed Engl. 2012; 51:11122-25. https://doi.org/10.1002/anie.201204169.

14. Lomas H, Johnston AP, Such GK, Zhu Z, Liang K, van Koeverden MP, Alongkornchotikul S, Caruso F. Polymersome-loaded capsules for controlled release of DNA. Small. 2011; 7:2109-19. https://doi.org/10.1002/ smll.201100744.

15. Canton I, Battaglia G. Polymersomes-mediated delivery of fluorescent probes for targeted and long-term imaging in live cell microscopy. Methods Mol Biol. 2013; 991:343-51. https://doi.org/10.1007/978-1-62703-336-7_31.

16. Chierico L, Joseph AS, Lewis AL, Battaglia G. Live cell imaging of membrane/cytoskeleton interactions and membrane topology. Sci Rep. 2014; 4:6056. https://doi. org/10.1038/srep06056.

17. Simón-Gracia L, Hunt H, Scodeller P, Gaitzsch J, Kotamraju VR, Sugahara KN, Tammik O, Ruoslahti E, Battaglia G, Teesalu T. iRGD peptide conjugation potentiates intraperitoneal tumor delivery of paclitaxel with polymersomes. Biomaterials. 2016; 104:247-57. https://doi. org/10.1016/j.biomaterials.2016.07.023.

18. Hunt H, Simón-Gracia L, Tobi A, Kotamraju VR, Sharma S, Nigul M, Sugahara KN, Ruoslahti E, Teesalu T. Targeting of p32 in peritoneal carcinomatosis with intraperitoneal linTT1 peptide-guided pro-apoptotic nanoparticles. J Control Release. 2017; 260:142-53. https://doi.org/10.1016/j. jconrel.2017.06.005.

19. Tian X, Nyberg S, S Sharp P, Madsen J, Daneshpour N, Armes SP, Berwick J, Azzouz M, Shaw P, Abbott NJ, Battaglia G. LRP-1-mediated intracellular antibody delivery to the Central Nervous System. Sci Rep. 2015; 5:11990. https://doi.org/10.1038/srep11990.

20. Smith TT, Stephan SB, Moffett HF, McKnight LE, Ji W, Reiman D, Bonagofski E, Wohlfahrt ME, Pillai SP, Stephan MT. In situ programming of leukaemia-specific T cells using synthetic DNA nanocarriers. Nat Nanotechnol. 2017; 12:813-20. https://doi.org/10.1038/nnano.2017.57.

21. Teesalu T, Sugahara KN, Ruoslahti E. Tumor-penetrating peptides. Front Oncol. 2013; 3:216. https://doi.org/10.3389/ fonc.2013.00216.

22. Sugahara KN, Teesalu T, Karmali PP, Kotamraju VR, Agemy L, Girard OM, Hanahan D, Mattrey RF, Ruoslahti E. Tissue-penetrating delivery of compounds and nanoparticles into tumors. Cancer Cell. 2009; 16:510-20. https://doi.org/10.1016/j.ccr.2009.10.013.

23. Paasonen L, Sharma S, Braun GB, Kotamraju VR, Chung TD, She ZG, Sugahara KN, Yliperttula M, Wu B, Pellecchia M, Ruoslahti E, Teesalu T. New p32/gC1qR Ligands for Targeted Tumor Drug Delivery. ChemBioChem. 2016; 17:570-75. https://doi.org/10.1002/cbic.201500564.

24. Fogal V, Zhang L, Krajewski S, Ruoslahti E. Mitochondrial/ cell-surface protein $\mathrm{p} 32 / \mathrm{gC} 1 \mathrm{qR}$ as a molecular target in tumor cells and tumor stroma. Cancer Res. 2008; 68:7210 18. https://doi.org/10.1158/0008-5472.CAN-07-6752.

25. Yenugonda V, Nomura N, Kouznetsova V, Tsigelny I, Fogal V, Nurmemmedov E, Kesari S, Babic I. A novel small molecule inhibitor of p32 mitochondrial protein overexpressed in glioma. J Transl Med. 2017; 15:210. https://doi.org/10.1186/s12967-017-1312-7.

26. Agemy L, Kotamraju VR, Friedmann-Morvinski D, Sharma $\mathrm{S}$, Sugahara KN, Ruoslahti E. Proapoptotic peptidemediated cancer therapy targeted to cell surface p32. Mol Ther. 2013; 21:2195-204. https://doi.org/10.1038/ mt.2013.191.

27. Sharma S, Kotamraju VR, Mölder T, Tobi A, Teesalu T, Ruoslahti E. Tumor-penetrating nanosystem strongly suppresses breast tumor growth. Nano Lett. 2017; 17:13561364. https://doi.org/10.1021/acs.nanolett.6b03815.

28. Braun GB, Sugahara KN, Yu OM, Kotamraju VR, Mölder T, Lowy AM, Ruoslahti E, Teesalu T. Urokinase-controlled tumor penetrating peptide. J Control Release. 2016; 232:188-95. https://doi.org/10.1016/j.jconrel.2016.04.027.

29. Ahrens ET, Bulte JW. Tracking immune cells in vivo using magnetic resonance imaging. Nat Rev Immunol. 2013; 13:755-63. https://doi.org/10.1038/nri3531.

30. Puaux AL, Ong LC, Jin Y, Teh I, Hong M, Chow PK, Golay $\mathrm{X}$, Abastado JP. A comparison of imaging techniques to monitor tumor growth and cancer progression in living animals. Int J Mol Imaging. 2011; 2011:321538. https://doi. org/10.1155/2011/321538.

31. Domingos RF, Baalousha MA, Ju-Nam Y, Reid MM, Tufenkji N, Lead JR, Leppard GG, Wilkinson KJ. Characterizing manufactured nanoparticles in the 
environment: multimethod determination of particle sizes. Environ Sci Technol. 2009; 43:7277-84. https://doi. org/10.1021/es900249m.

32. Irigoyen J, Arekalyan VB, Navoyan Z, Iturri J, Moya SE, Donath E. Spherical polyelectrolyte brushes' constant zeta potential with varying ionic strength: an electrophoretic study using a hairy layer approach. Soft Matter. 2013; 9:11609. https://doi.org/10.1039/c3sm51343b.

33. Scodeller P, Simón-Gracia L, Kopanchuk S, Tobi A, Kilk K, Säälik P, Kurm K, Squadrito ML, Kotamraju VR, Rinken A, De Palma M, Ruoslahti E, Teesalu T. Precision targeting of tumor macrophages with a CD206 binding peptide. Sci Rep. 2017; 7:14655. https://doi.org/10.1038/ s41598-017-14709-x.

34. Fogal V, Richardson AD, Karmali PP, Scheffler IE, Smith JW, Ruoslahti E. Mitochondrial p32 protein is a critical regulator of tumor metabolism via maintenance of oxidative phosphorylation. Mol Cell Biol. 2010; 30:1303-18. https:// doi.org/10.1128/MCB.01101-09. Erratum in: Correction for Fogal et al., "Mitochondrial p32 Protein Is a Critical Regulator of Tumor Metabolism via Maintenance of Oxidative Phosphorylation". [Mol Cell Biol. 2017].

35. DeNardo DG, Barreto JB, Andreu P, Vasquez L, Tawfik D, Kolhatkar N, Coussens LM. CD4(+) T cells regulate pulmonary metastasis of mammary carcinomas by enhancing protumor properties of macrophages. Cancer Cell. 2009; 16:91-102. https://doi.org/10.1016/j. ccr.2009.06.018.

36. Martinez-Pomares L. The mannose receptor. J Leukoc Biol. 2012; 92:1177-86. https://doi.org/10.1189/jlb.0512231.

37. Lewis CE, Harney AS, Pollard JW. The multifaceted role of perivascular macrophages in tumors. Cancer Cell. 2016; 30:18-25. https://doi.org/10.1016/j.ccell.2016.05.017.

38. Phillips E, Penate-Medina O, Zanzonico PB, Carvajal RD, Mohan P, Ye Y, Humm J, Gönen M, Kalaigian H, Schöder H, Strauss HW, Larson SM, Wiesner U, Bradbury MS. Clinical translation of an ultrasmall inorganic opticalPET imaging nanoparticle probe. Sci Transl Med. 2014; 6:260ra149. https://doi.org/10.1126/scitranslmed.3009524.

39. Longmire M, Choyke PL, Kobayashi H. Clearance properties of nano-sized particles and molecules as imaging agents: considerations and caveats. Nanomedicine (Lond). 2008; 3:703-17. https://doi.org/10.2217/17435889.3.5.703.

40. Grossen P, Witzigmann D, Sieber S, Huwyler J. PEG-PCLbased nanomedicines: A biodegradable drug delivery system and its application. J Control Release. 2017; 260:46-60. https://doi.org/10.1016/j.jconrel.2017.05.028.

41. Kim SC, Kim DW, Shim YH, Bang JS, Oh HS, Wan Kim $\mathrm{S}$, Seo MH. In vivo evaluation of polymeric micellar paclitaxel formulation: toxicity and efficacy. J Control Release. 2001; 72:191-202. https://doi.org/10.1016/ S0168-3659(01)00275-9.
42. Benito AB, Aiertza MK, Marradi M, Gil-Iceta L, Shekhter Zahavi T, Szczupak B, Jiménez-González M, Reese T, Scanziani E, Passoni L, Matteoli M, De Maglie M, Orenstein A, et al. Functional single-chain polymer nanoparticles: targeting and imaging pancreatic tumors in vivo. Biomacromolecules. 2016; 17:3213-21. https://doi. org/10.1021/acs.biomac.6b00941.

43. Lee H, Hoang B, Fonge H, Reilly RM, Allen C. In vivo distribution of polymeric nanoparticles at the whole-body, tumor, and cellular levels. Pharm Res. 2010; 27:2343-55. https://doi.org/10.1007/s11095-010-0068-z.

44. Pérez-Medina C, Abdel-Atti D, Tang J, Zhao Y, Fayad ZA, Lewis JS, Mulder WJ, Reiner T. Nanoreporter PET predicts the efficacy of anti-cancer nanotherapy. Nat Commun. 2016; 7:11838. https://doi.org/10.1038/ncomms11838.

45. Ruoslahti E. Tumor penetrating peptides for improved drug delivery. Adv Drug Deliv Rev. 2017; 110-111:3-12. https:// doi.org/10.1016/j.addr.2016.03.008.

46. Mekkawy AH, Pourgholami MH, Morris DL. Involvement of urokinase-type plasminogen activator system in cancer: an overview. Med Res Rev. 2014; 34:918-56. https://doi. org/10.1002/med.21308.

47. Teesalu T, Sugahara KN, Kotamraju VR, Ruoslahti E. $\mathrm{C}$-end rule peptides mediate neuropilin-1-dependent cell, vascular, and tissue penetration. Proc Natl Acad Sci U S A. 2009; 106:16157-62. https://doi.org/10.1073/ pnas.0908201106.

48. Hussain S, Rodriguez-Fernandez M, Braun GB, Doyle FJ, Ruoslahti E. Quantity and accessibility for specific targeting of receptors in tumours. Sci Rep. 2014; 4:5232. https://doi. org/10.1038/srep05232.

49. Miller MA, Zheng YR, Gadde S, Pfirschke C, Zope H, Engblom C, Kohler RH, Iwamoto Y, Yang KS, Askevold B, Kolishetti N, Pittet M, Lippard SJ, et al. Tumour-associated macrophages act as a slow-release reservoir of nanotherapeutic Pt(IV) pro-drug. Nat Commun. 2015; 6:8692. https://doi.org/10.1038/ncomms9692.

50. Zhang F, Niu G, Lin X, Jacobson O, Ma Y, Eden HS, He Y, Lu G, Chen X. Imaging tumor-induced sentinel lymph node lymphangiogenesis with LyP-1 peptide. Amino Acids. 2012; 42:2343-51. https://doi.org/10.1007/s00726-011-0976-1.

51. Wallace AM, Hoh CK, Limmer KK, Darrah DD, Schulteis G, Vera DR. Sentinel lymph node accumulation of Lymphoseek and Tc-99m-sulfur colloid using a "2-day" protocol. Nucl Med Biol. 2009; 36:687-92. https://doi. org/10.1016/j.nucmedbio.2009.04.007.

52. Hamzah J, Kotamraju VR, Seo JW, Agemy L, Fogal V, Mahakian LM, Peters D, Roth L, Gagnon MK, Ferrara KW, Ruoslahti E. Specific penetration and accumulation of a homing peptide within atherosclerotic plaques of apolipoprotein E-deficient mice. Proc Natl Acad Sci U S A. 2011; 108:7154-59. https://doi.org/10.1073/pnas.1104540108. 